\title{
Progenitor identification and SARS-CoV-2 infection in human distal lung organoids
}

https://doi.org/10.1038/s41586-020-3014-1

Received: 8 November 2017

Accepted: 18 November 2020

Published online: 25 November 2020

Check for updates

\author{
Ameen A. Salahudeen ${ }^{1,19,20}$, Shannon S. Choi ${ }^{1,20}$, Arjun Rustagi ${ }^{2}$, Junjie Zhu ${ }^{3}$, \\ Vincent van Unen ${ }^{4,5}$, Sean M. de la O', Ryan A. Flynn ${ }^{6,7}$, Mar Margalef-Català ${ }^{8}$, \\ António J. M. Santos' ${ }^{1}$ Jihang Ju', Arpit Batish', Tatsuya Usui', Grace X. Y. Zheng ${ }^{9}$, \\ Caitlin E. Edwards ${ }^{10}$, Lisa E. Wagar ${ }^{4,5}$, Vincent Luca", Benedict Anchang ${ }^{12}$, \\ Monica Nagendran ${ }^{13}$, Khanh Nguyen ${ }^{14}$, Daniel J. Hart', Jessica M. Terry ${ }^{9}$, Phillip Belgrader ${ }^{9}$, \\ Solongo B. Ziraldo ${ }^{9}$, Tarjei S. Mikkelsen ${ }^{9}$, Pehr B. Harbury ${ }^{15}$, Jeffrey S. Glenn ${ }^{4,14}$, \\ K. Christopher Garciaa, ${ }^{11,16}$, Mark M. Davis ${ }^{4,5,16}$, Ralph S. Baric ${ }^{10,17}$, Chiara Sabatti ${ }^{12}$, \\ Manuel R. Amieva ${ }^{4,8}$, Catherine A. Blish ${ }^{2,18 \bowtie}$, Tushar J. Desai ${ }^{13 \bowtie}$ \& Calvin J. Kuo ${ }^{1 凶}$
}

\begin{abstract}
The distal lung contains terminal bronchioles and alveoli that facilitate gas exchange. Three-dimensional in vitro human distal lung culture systems would strongly facilitate the investigation of pathologies such as interstitial lung disease, cancer and coronavirus disease 2019 (COVID-19) pneumonia caused by severe acute respiratory syndrome coronavirus 2 (SARS-CoV-2). Here we describe the development of a long-term feeder-free, chemically defined culture system for distal lung progenitors as organoids derived from single adult human alveolar epithelial type II (AT2) or KRT5 ${ }^{+}$ basal cells. AT2 organoids were able to differentiate into AT1 cells, and basal cell organoids developed lumens lined with differentiated club and ciliated cells. Single-cell analysis of $K R T 5^{+}$cells in basal organoids revealed a distinct population of $I T G A 6^{+} I T G B 4^{+}$mitotic cells, whose offspring further segregated into a TNFRSF $12 A^{h i}$ subfraction that comprised about ten per cent of KRT $5^{+}$basal cells. This subpopulation formed clusters within terminal bronchioles and exhibited enriched clonogenic organoid growth activity. We created distal lung organoids with apical-out polarity to present ACE2 on the exposed external surface, facilitating infection of AT2 and basal cultures with SARS-CoV-2 and identifying club cells as a target population. This long-term, feeder-free culture of human distal lung organoids, coupled with single-cell analysis, identifies functional heterogeneity among basal cells and establishes a facile in vitro organoid model of human distal lung infections, including COVID-19-associated pneumonia.
\end{abstract}

The distal lung performs essential respiratory functions that can be compromised by inflammatory, neoplastic or infectious disorders such as COVID-19 pneumonia. The study of these conditions would be facilitated by robust in vitro models based on human cells. The identities of the stem cells that regenerate distal lung epithelium in vivo over the human lifespan have been inferred from mouse studies, despite differences between these species ${ }^{1}$. In humans, basal stem cells span the entire airway tree, whereas in mouse, club cells ${ }^{2}$ and/or secretoglobin $1 \mathrm{~A} 1$ (SCGB1A1)-expressing non-club cells ${ }^{3}$ renew the distal bronchioles during ageing. In the gas exchange region, mouse alveolar epithelial type II (AT2) cells generate AT1 and AT 2 cells during homeostasis ${ }^{4,5}$, and additional progenitors are recruited in response to severe injury $\mathrm{y}^{3,6-9}$. The presence and/or roles of facultative progenitors in human lung are unknown. Human AT2 cells can be differentiated into AT1 cells, but these cultures are short-lived and do not demonstrate long-term self-renewal or enable expansion for disease modelling ${ }^{4,10,11}$; furthermore, their requirements for feeder cells impede the definition of specific niche components ${ }^{12,13}$. We have established long-term organoid culture of distal human lung,

'Division of Hematology, Department of Medicine, Stanford University School of Medicine, Stanford, CA, USA. 'Division of Infectious Disease and Geographic Medicine, Department of Medicine, Stanford University School of Medicine, Stanford, CA, USA. ${ }^{3}$ Stanford University School of Engineering, Department of Electrical Engineering, Stanford, CA, USA. ${ }^{4}$ Department of Microbiology and Immunology, Stanford University School of Medicine, Stanford, CA, USA. ${ }^{5}$ Stanford Institute of Immunity, Transplantation and Infection, Stanford University School of

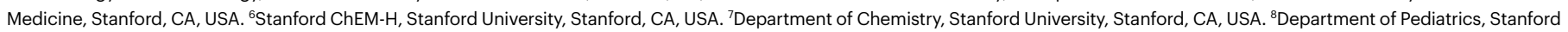
University School of Medicine, Stanford, CA, USA. ${ }^{9} 10 x$ Genomics, Pleasanton, CA, USA. ${ }^{10}$ Department of Epidemiology, University of North Carolina at Chapel Hill, Chapel Hill, NC, USA. ${ }^{11}$ Department of Molecular and Cellular Physiology, Stanford University School of Medicine, Stanford, CA, USA. ${ }^{12}$ Division of Biomedical Data Science, Department of Medicine, Stanford University School of Medicine, Stanford, CA, USA. ${ }^{13}$ Division of Pulmonary, Allergy and Critical Care, Department of Medicine, Stanford University School of Medicine, Stanford, CA, USA. ${ }^{14}$ Division of Gastroenterology, Department of Medicine, Stanford University School of Medicine, Stanford, CA, USA. ${ }^{15}$ Department of Biochemistry, Stanford University School of Medicine,

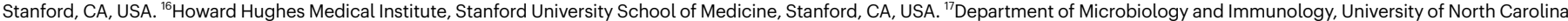

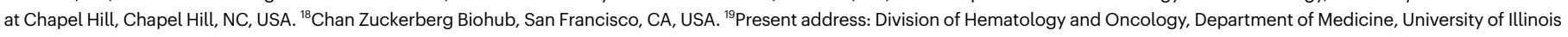
at Chicago College of Medicine, Chicago, IL, USA. ${ }^{20}$ These authors contributed equally: Ameen A. Salahudeen, Shannon S. Choi. ${ }^{凶 e-m a i l: ~ c b l i s h @ s t a n f o r d . e d u ; ~ t d e s a i @ s t a n f o r d . e d u ; ~}$ cjkuo@stanford.edu 

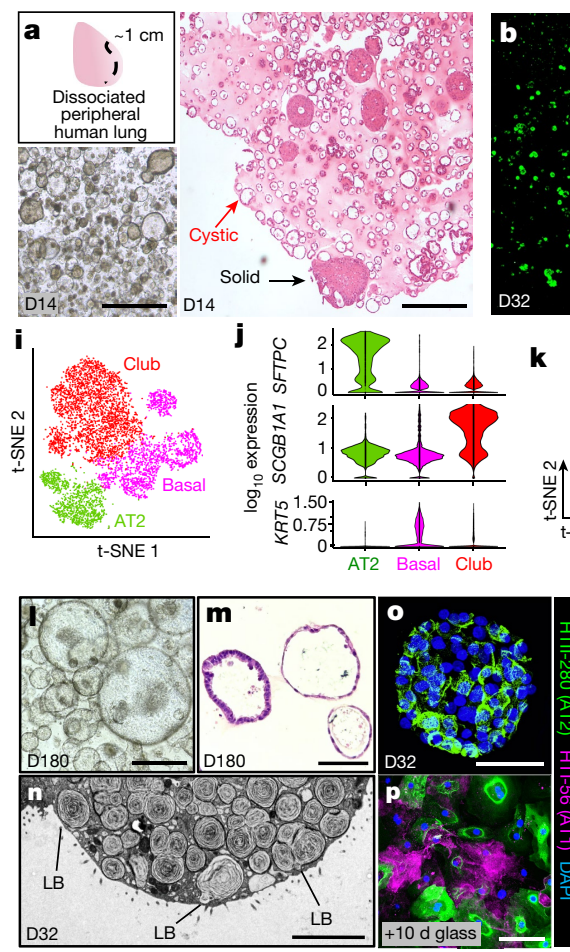

Fig. 1 | Clonogenic expansion of human distal lung organoids. a. Human distal lung D14 (day 14) organoid cultures contain cystic and solid organoids. Bottom left, brightfield; right, haematoxylin and eosin (H\&E). Scale bar, $100 \mu \mathrm{m} . \mathbf{b}$, Whole-mount immunofluorescence with anti-KRT5 (basal cell), anti-SFTPC (AT2 cell) and anti-SCGB1A1 (club cell) antibodies. Scale bar, $100 \mu \mathrm{m} . \mathbf{c}-\mathbf{e}$, Alveolar organoids on D32.c, Cystic AT2 organoid. H\&E; scale bar, $25 \mu \mathrm{m}$. d, Whole-mount immunofluorescence for anti-SFTPC, anti-HTII-280, phalloidin (Ph) and DAPI; scale bar, $50 \mu \mathrm{m}$.e, Anti-KI67 and DAPI fluorescence of an adjacent section to d. f-h, Basal organoids on D32.f, H\&E; scale bar, $50 \mu \mathrm{m}$. g, Whole-mount immunofluorescence for anti-KRT5 and DAPI; scale bar, $100 \mu \mathrm{m} . \mathbf{h}$, Anti-KI67 and DAPI immunostaining of adjacent section to $\mathbf{g}$. $\mathbf{i}-\mathbf{k}$, Single-cell RNA-seq of total distal lung organoids at D28.i, $t$-Distributed

including AT2 and basal stem cells, and used this system to validate functional progenitor cells and model SARS-CoV-2 infection.

\section{Clonogenic alveolar and basal organoids}

We empirically defined medium conditions to support the clonal expansion of distal human lung progenitors from 136 individuals in collagen/laminin extracellular matrix (ECM) (Fig. 1a, Supplementary Table 1). Together, EGF and the BMP antagonist NOGGIN supported growth of disaggregated distal lung cells or purified epithelial fractions thereof (Extended Data Fig. 1a-c). Single cells (Extended Data Fig. 1d-g) expanded into either SFTPC ${ }^{+}$HTII- $280^{+}$AT2 cystic organoids (Fig. 1b-e) or KRT5 ${ }^{+}$solid organoids expressing the basal cell marker KRT5 (Fig. 1b, f-h).

Single cell RNA sequencing (scRNA-seq) of distal lung organoids confirmed the presence of populations of $S F T P C^{+} \mathrm{AT} 2$ cells, $K R T 5^{+}$basal cells and $S C G B 1 A 1^{+}$club cells (Fig. 1i,j, Extended Data Fig. 2 ). Cells that co-expressed KRT5 and SCGB1A1 were found in both basal and club cell populations, suggesting a transitional state (Fig. 1j, k, Extended Data Fig. 2) that was supported by the results of SPADE ${ }^{14}$ and Monocle ${ }^{15}$ trajectory analysis (Extended Data Fig. 3, Supplementary Table 2).

\section{Characterization of human AT2 cell organoids}

We generated pure AT2 organoids from mixed cultures via uptake of lysosomal dyes into lamellar bodies (Extended Data Fig. 4a, b,
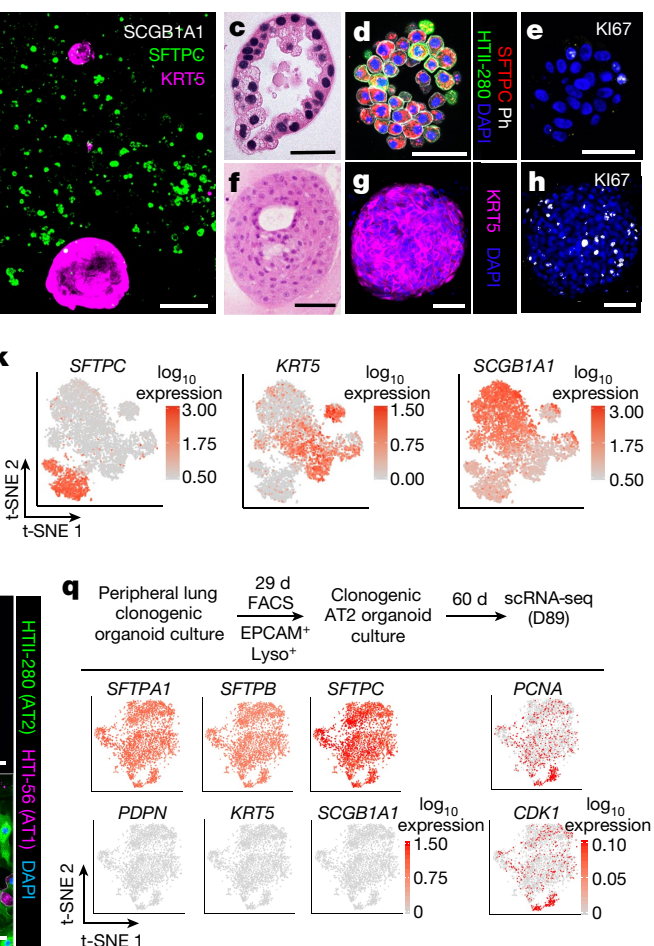

stochastic neighbour embedding (t-SNE) plot of 7,285 individual cells demonstrating AT2, basal, and club populations.j, Expression of SFTPC (AT2), $K R T 5$ (basal) and SCGB1A1 (club). k, Feature plots for expression of SFTPC (AT2), $K R T 5$ (basal) and SCGB1A1 (club). I-p, Clonogenic AT2 organoid culture. I, Brightfield microscopy of AT2 organoids at D180; scale bar, $200 \mu \mathrm{m}$. m, H\&E staining from culture as in $\mathbf{l}$; scale bar, $50 \mu \mathrm{m}$. $\mathbf{n}$, Transmission electron microscopy of AT2 organoid at D32. LB, lamellar body; scale bar, $5 \mu \mathrm{m}$. $\mathbf{o , p}$, Immunofluorescence of AT2 organoid at D32 (o); culture on glass for 10 additional days induces differentiation into AT1 cells (p). Immunofluorescence for anti-HTI-56 (AT1) and anti-HTII-280 (AT2); scale bar, $50 \mu \mathrm{m}$. q, scRNA-seq feature plots of 2,780 AT2 organoid cells on D89 of cumulative culture.

Supplementary Fig. 1). EPCAM ${ }^{+}$LysoTracker ${ }^{+}$AT2 cells expanded clonally for up to 180 days (Fig. 1l, m, Extended Data Fig. 4c, d). Chemically defined EGF/NOGGIN medium was sufficient for baseline clonal proliferation of AT2 organoids, which was attenuated by blocking global endogenous WNT biosynthesis (Extended Data Fig. 4e), consistent with the requirement for autocrine WNT signalling in mouse AT 2 cells ${ }^{16}$. Growth was enhanced by adding fibroblast conditioned medium containing serum and WNT agonists (Extended Data Fig. 4f). Transmission electron microscopy revealed microvilli and lamellar bodies characteristic of mature AT2 cells (Fig. 1n, Extended Data Fig. 4g). AT2 organoids showed upregulation of AT1 markers when cultured on glass with serum (Fig. 1o, p).

Single-cell RNA-seq of mixed distal lung organoids or purified alveolar organoids revealed uniformly high expression of canonical AT2 cell markers in alveolar populations (Fig. 1i-k, q, Extended Data Fig. 2, Supplementary Data 1$)$. AT 2 cell subsets were not readily observed, and cell cycle-related mRNAs did not localize to a specific AT2 subpopulation (Fig. 1q, Supplementary Data 1, Supplementary Table 3).

\section{Differentiation of human basal organoids}

Basal cell organoids in mixed distal lung culture grew more rapidly than alveolar organoids and initially formed solid $\mathrm{KRT}^{+}$spheroids (Fig. 1a, b, f-h). However, by one month of culture, about $50 \%$ of organoids had developed single or occasionally multiple lumens (Fig. 2a,b, Extended Data Fig. 5a-c) with club (SCGB1A1 ${ }^{+} \mathrm{KRT}^{-}$) and ciliated 


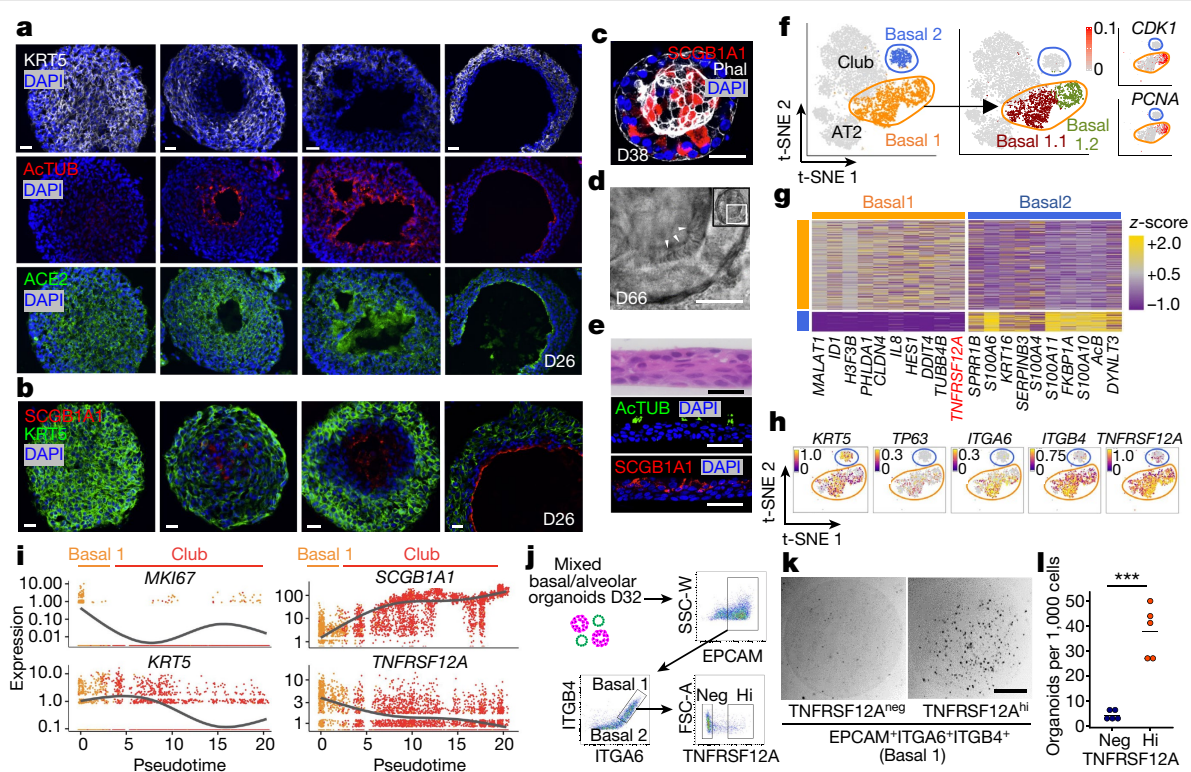

Fig. 2 | Basal organoid differentiation and TNFRSF12A ${ }^{\text {hi }}$ progenitor discovery. a, b, Immunofluorescence showing basal organoid differentiation in mixed culture. Lumens are lined with acetylated tubulin ${ }^{+}$(AcTUB) ciliated cells, which express the SARS-CoV-2 receptor ACE2 but lack KRT5 (a), and SCGB1A1 ${ }^{+}$club cells (b). Scale bar, $20 \mu \mathrm{m}$. c-e, Sedimented basal organoid culture. c, Club cell differentiation in purified basal organoids after 38 days in 3D clonogenic culture, shown by whole-mount fluorescence for anti-SCGB1A1 (red), phalloidin (white), and DAPI (blue); scale bar, $50 \mu \mathrm{m}$. d, Ciliated differentiation of organoids as in c. Confocal transmission image; scale bar, $20 \mu \mathrm{m}$. White box in inset expanded to main image; arrowheads denote cilia. e, Immunofluorescence showing $\mathrm{AcTUB}^{+}$ciliated and $\mathrm{SCGB}^{\mathrm{A}} \mathrm{1}^{+}$club cells in an organoid that has been replated in 2D ALI culture. Scale bar, $50 \mu \mathrm{m}$.

(AcTUB ${ }^{+} \mathrm{KRT}^{-}$) cells lining the interior surface (Fig. 2a, b). Basal cultures purified by density sedimentation exhibited serial clonal outgrowth, dependence on EGF and NOGGIN, and cavitation lined by luminal SCGB1A1 ${ }^{+}$club and AcTUB ${ }^{+}$ciliated cells (Fig. 2c, d, Extended Data Fig. 5d-h, Supplementary Videos 1, 2). Similar differentiation occurred when organoids were transferred into 2D air-liquid interface (ALI) cultures (Fig. 2e).

\section{Distinct subtypes of airway basal cells}

Single-cell RNA-seq clustering of organoid $K R T 5^{+}$basal cells from multiple individuals reproducibly identified two populations: Basal 1 and Basal 2 (Fig. 2f, g, Extended Data Fig. 6a, Supplementary Data 1). Basal 1 included an actively cycling subpopulation (Basal 1.2) that was enriched in proliferation markers ( $P C N A, C D K 1)$ and gene cell enrichment analysis (GSEA) cell-cycle-related processes (Fig. 2f, g, Extended Data Fig. 6a-c, Supplementary Table 3). Basal 1, but not Basal 2, expressed canonical lung basal cell mRNAs such as TP6 $3^{17}$, integrin- $\alpha_{6}(I T G A 6)$ and integrin- $\beta_{4}$ (ITGB4, which encodes a binding partner of ITGA $6^{18}$ and marks mouse lineage-negative epithelial progenitors (LNEPs) $)^{7}$ (Fig. 2h). Basal 2 was enriched in expression of genes related to vesicular transport, endoplasmic reticulum processes and squamous markers (Fig. 2g, Supplementary Table 3).

\section{Characterization of TNFRSF12A $\mathrm{A}^{+}$progenitors}

We examined the differentially expressed Basal 1 gene TNFRSF12A (also known as Fn14, TWEAKR), which encodes a membrane receptor (Fig. 2g, h, Supplementary Table 4) because of its potential utility for fluorescence-activated cell sorting (FACS) isolation and its homology to f, g, Single-cell RNA-seq of $K R T 5^{+}$basal cells from Fig. 1i.f, t-SNE plots showing Basal 1 and Basal 2 subclusters. Basal 1 is subdivided into Basal 1.1 and 1.2, with the latter expressing the proliferative mRNAs $C D K 1$ and $P C N A$.g, Differential gene expression in Basal 1 and Basal 2 subclusters from a total of 2,303 basal cells. h, Basal marker transcript t-SNE overlays of $\mathbf{f}\left(\log _{10}\right.$ number of unique molecular identifiers (UMI)). i, Relative gene expression kinetic plots of $\mathbf{f}$ and $\mathbf{g}$ across the pseudotime trajectory ( $n=3,721$ cells). $\mathbf{j}$, FACS isolation of TNFRSF12 $^{\text {hi }}$ versus TNFRSF12 ${ }^{\text {neg }}$ cells from mixed distal lung organoids, pre-gated on live singlets. $\mathbf{k}$, Brightfield image of organoid culture of cells from j at D14. I, Quantification of $\mathbf{k}$ (number of organoids per 1,000 cells); ${ }^{* * *} P<0.001$ $\left(P=1.0 \times 10^{-4}\right)$, two-tailed Student's $t$-test. Data in $\mathbf{j}, \mathbf{k}$ represent $n=5$ independent experiments for each TNFRSF12A population.

the intestinal stem cell marker TNFRSF19 ${ }^{19}$. Unbiased pseudotime analysis revealed a continuous single-cell trajectory connecting $K R T 5^{+}$Basal 1 cells to $S C G B 1 A 1^{+}$club cells, with $T N F R S F 12 A$ mRNA being strongly associated with the proliferation marker gene $M K I 67$ (Fig. 2i, Extended Data Fig. 3e). When Basal 1 cells co-expressing EPCAM, ITGA6 and ITGB4 were divided into three fractions (expressing low, medium and high levels of TNFRSF12A mRNA), a proliferative gene modul ${ }^{20}$ was significantly enriched in the highest ( TNFRSF12 $A^{\text {hi }}$ ) versus lowest fraction $\left(\right.$ TNFRSF12A $\left.A^{l o}\right)$ (Extended Data Fig. 6d-f). To determine whether this enrichment reflected intrinsic proliferative potential, we fractionated total distal lung organoids by FACS into EPCAM ${ }^{+}$ITGA $^{+}{ }^{+}$TGB $^{+}$Basal 1 cells and then into TNFRSF12 ${ }^{\text {hi }}$ and TNFRSF12 ${ }^{\text {neg }}$ subsets (Fig. $2 \mathrm{j}$ ). When cultured, the TNFRSF12 $\mathrm{A}^{\text {hi }}$ subset showed 4-12 times greater clonogenic organoid-forming capacity than the TNFRSF $12 A^{\text {neg }}$ subset (Fig. 2k, l).

We examined lineage relationships between Basal 1 and Basal 2 by fractionating density-sedimented $\mathrm{KRT}^{+}$basal organoids by FACS into EPCAM ${ }^{+}$ITGA $^{+}$ITGB $^{+}{ }^{+}$TNFRSF12A ${ }^{\text {hi }}$ (Basal 1 ) and EPCAM $^{+}$ITGA6 $^{-}$ITGB4-TNFRSF12A $^{\text {neg }}$ (Basal 2) populations (Extended Data Fig. 7a).Clonogenic organoid formation was strongly enriched in Basal 1 versus Basal 2 from three separate individuals (Extended Data Fig. 7b-d). The basal 2-enriched genes SPRR1B and TMSB4X (Fig. 2g, Supplementary Table 3) were transiently induced in Basal 1 cell organoids (Extended Data Fig. 7e, f), suggesting that Basal 2 cells might differentiate from Basal 1.

The NOTCH target gene HES1 was one of the most differentially expressed loci in Basal 1 organoids, in which gene networks included NOTCH1, NOTCH2 and JAG1 (Fig. 2g, Supplementary Table 3). Inhibition of NOTCH significantly increased basal organoid proliferation from TNFRSF12A $^{\text {hi }}$ EPCAM $^{+}$ITGA6 $^{+}$ITGB4 $^{+}$cells (Extended Data Fig. 7g, h), 
a

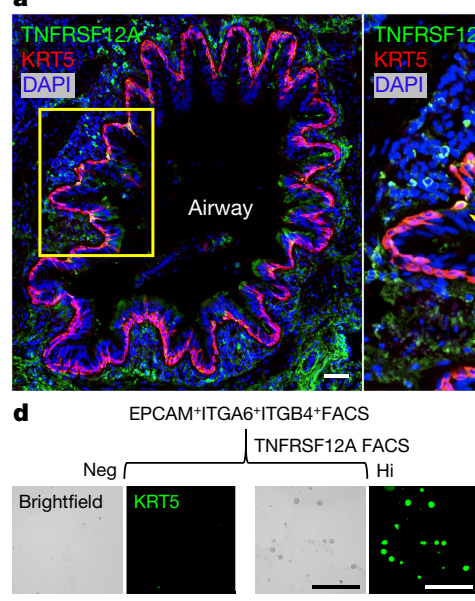

g

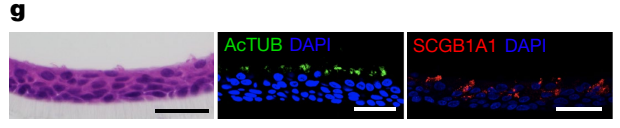

b

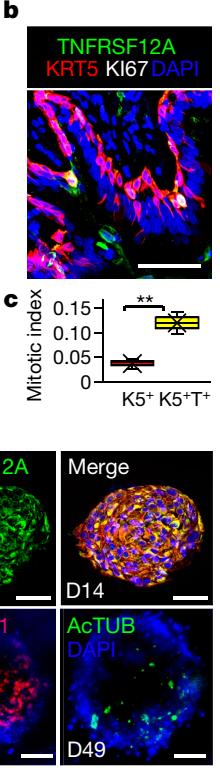

Fig. 3 | Characterization of TNFRSF12 $A^{\text {hi }}$ Basal 1 cells from intact human lung. a, Left, anti-KRT5 and anti-TNFRSF12A immunofluorescence of human distal lung. Yellow boxed area is expanded with (middle) and without (right) DAPI. Scale bars, $100 \mu \mathrm{m}$. b. Immunofluorescence of distal airway TNFRSF $12 \mathrm{~A}^{+}$ basal cell proliferation showing KRT5, TNFRSF12A, KI67 and DAPI. Scale bar, $100 \mu \mathrm{m} . \mathbf{c}$, Mitotic index of TNFRSF $12 \mathrm{~A}^{+} \mathrm{KRTS}^{+}$cells from b; three independent experiments. $\mathrm{K}^{+}$, total $\mathrm{KRT}^{+} ; \mathrm{K} 5^{+} \mathrm{T}^{+}$, TNFRSF $12 \mathrm{~A}^{+} \mathrm{KRT} 5^{+}$. Box plots represent first quartile, median and third quartile; whiskers show minimum and maximum. ${ }^{* *} P<0.01\left(P=4.4 \times 10^{-3}\right)$, two-tailed Student's $t$-test. d, Brightfield and anti-KRT5 immunofluorescence of FACS-isolated TNFRSF12A $\mathrm{A}^{\text {neg }}$ and

suggesting that NOTCH signalling restrains growth in these cells. Conversely, NOTCH agonism did not affect proliferation, but did induce expression of SCGB1A1, similar to upper airway cells ${ }^{21,22}$ (Extended Data Fig. 7i).

\section{Characterization of TNFRSF12A $A^{+}$cells in distal lung}

Immunostaining of human distal lung tissue showed TNFRSF12A ${ }^{+}$ basal cells that were enriched intermittently at the tips or bases of bronchiolar furrows (Fig. 3a, Extended Data Fig. 8a, b); the latter are recognized as a goblet cell niche ${ }^{23}$. We found TNFRSF12A in diverse lung stromal and epithelial cells, but it clearly marked a minor population

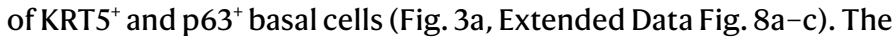
TNFRSF12A ${ }^{+}$subset of KRT $5^{+}$basal cells had a higher mitotic index than total KRT $5^{+}$cells in vivo (Fig. 3b, c), consistent with the results of organoid scRNA-seq (Fig. 2i). FACS analysis of human distal lung cells confirmed that TNFRSF12A was expressed in $10.9 \%$ of basal cells (Extended Data Fig. 8d, top).

Upon prospective culture directly from human lung without an organoid intermediate, FACS-isolated EPCAM $^{+}$ITGA $^{+}$ITGB $^{+}{ }^{+}$TNFRSF $12 A^{\text {hi }}$ cells (TNFRSF12A ${ }^{\text {hi }}$ Basal 1 cells; Extended Data Fig. 8d, bottom) showed a 15-fold increase in $\mathrm{KRT}^{+}$organoid formation compared with EPCAM $^{+}$ITGA6 $^{+}$ITGB $^{+}{ }^{+}$TNFRSF $12 A^{\text {neg }}$ cells (TNFRSF $12^{\text {neg }}$ Basal 1 cells; Fig. 3d, e). TNFRSF12A hi basal organoids also differentiated into $\mathrm{SCGB} \mathrm{Al}^{+}$club and $\mathrm{AcTUB}^{+}$ciliated cells in prolonged culture (Fig. $3 \mathrm{f}$ ) or when grown as 2D ALI monolayers (Fig. 3g).

\section{SARS-CoV-2 and H1N1 infection of organoids}

Influenza virus H1N1 avidly infected distal lung organoids, which also expressed influenza receptors (Extended Data Fig. 9a-d), similar to proximal airway organoids ${ }^{24,25}$. Infection of organoids with influenza
TNFRSF12A ${ }^{\text {hi }}$ organoid cultures at D14; scale bars, $500 \mu \mathrm{m}$. e, Quantification of d (number of organoids per 1,000 cells); data represent $n=5$ independent experiments for each TNFRSF12A population. Box plots represent first quartile, median and third quartile; whiskers show minimum and maximum. $P=1.1 \times 10^{-3}$, two-tailed Student's $t$-test.f, H\&E and anti-KRT5, anti-TNFRSF12A, anti-SCGB1A1, and anti-AcTUB immunostaining of organoids from the TNFRSF12A ${ }^{\text {hi }}$ fraction of FACS-sorted EPCAM ${ }^{+} I T G A 6^{+}{ }^{+}{ }^{2} G B 4^{+}$distal lung cells. Scale bars, $50 \mu \mathrm{m} . \mathrm{g}$, H\&E and anti-SCGB1A1 and anti-AcTUB immunof luorescence of 2D ALI cultures from basal cell organoids from TNFRSF12A ${ }^{\text {hi }}$ basal cells as in $\mathbf{f}$. Scale bars, $50 \mu \mathrm{m}$.

H1N1 PR8 was inhibited by nucleoside analogues, consistent with previous studies ${ }^{26}$ (Extended Data Fig. 9e), and screening of diverse antiviral compound classes in 48-well format revealed differential activity (Extended Data Fig. 9f), suggesting that this system could be used for scalable therapeutics discovery.

In COVID-19 pneumonia, severe SARS-CoV-2 infection of the distal lung induces alveolar damage and respiratory failure ${ }^{27}$. Organoid scRNA-seq at time points before ciliated differentiation identified expression of the SARS-CoV-2 receptor $A C E 2$ and processing protease TMPRSS2 mRNAs predominantly in club and AT 2 cells (Extended Data Fig. 10a), consistent with expression of ACE2 in KRT5- differentiated interior luminal cells (Fig. 2a). To facilitate access of SARS-CoV-2 to ACE2-expressing luminal cells, we adapted an apical-out suspension culture polarization method ${ }^{28}$ to distal lung organoids (Extended Data Fig. 10b). Within $48 \mathrm{~h}$ in suspension, organoids reorganized into apical-out epithelial spheroids with microvilli, apical junctions, and motile cilia facing the organoid exterior. Differentiation of outwardly oriented ciliated cells accelerated over five days and progressed over weeks (Fig. 4a, Extended Data Fig. 10c-f, Supplementary Video 3). Apical-out basal organoids also showed an increase in outwardly facing club cells with apical secretory granules (Fig. 4a, Extended Data Fig. 10g), and apical-out AT2 organoids showed differentiation into AT1 cells (Extended Data Fig. 10h-j). Crucially, in apical-out organoids, ACE2 localized to apical cell membranes on the external organoid surface (Fig. 4b, Extended Data Fig. 10k).

SARS-CoV-2 infected apical-out mixed distal lung organoids, with induction of unspliced SARS-CoV-2 genomic RNA reaching levels similar to those of the abundantly expressed U3 small nucleolar RNA (Fig. 4c, left). In addition, infected organoids showed replication-specific SARS-CoV-2 spliced subgenomic RNA (sgRNA; Fig. 4c, right) and production of infectious virions with VeroE6 cell plaque formation (35 PFU ml ${ }^{-1}$ from organoid lysates and $65 \mathrm{PFU} \mathrm{ml}^{-1}$ from organoid supernatants). 

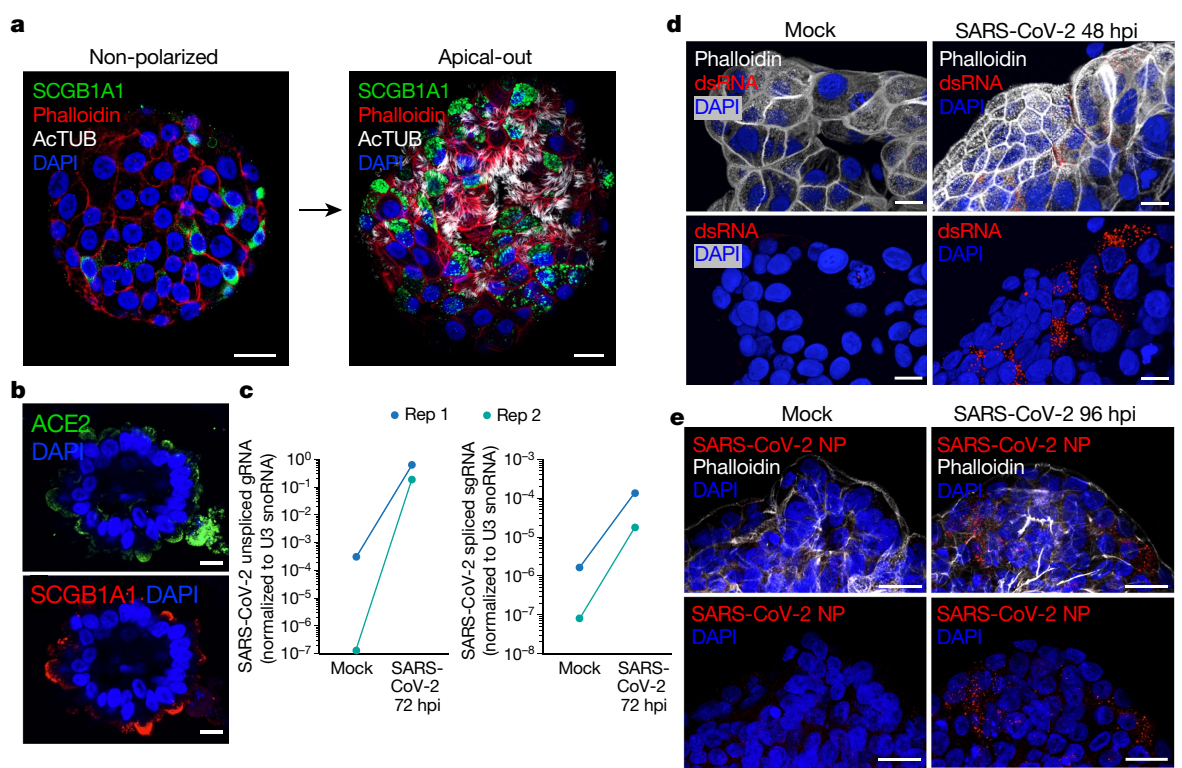

SARS-CoV-2 $96 \mathrm{hpi}$
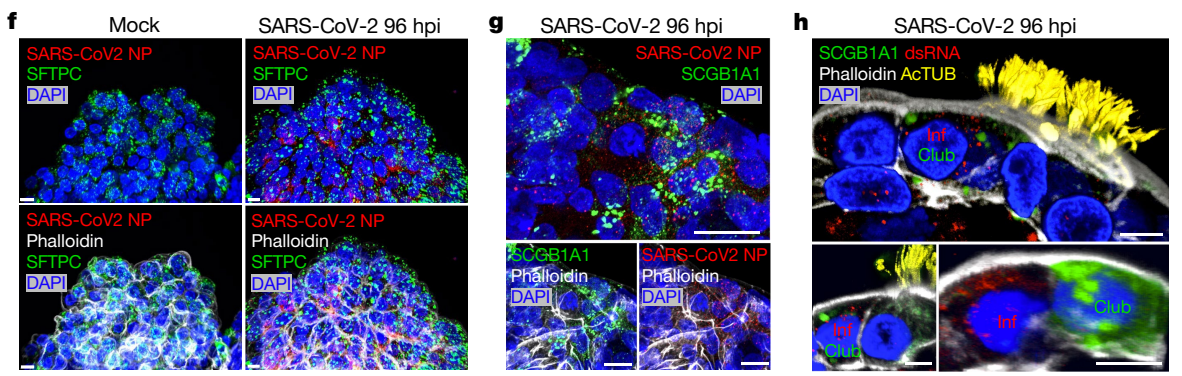

Fig. 4 | SARS-CoV-2 infection of apical-out distal lung organoids. a, Immunofluorescence comparing polarization and surface localization of SCGB1A1 ${ }^{+}$club cells (green) and $\mathrm{AcTUB}^{+}$ciliated cells (white) upon ECM-embedded non-polarized growth (left) or apical-out suspension culture (right).b, Transverse sections of apical-out basal organoid as in a stained with anti-ACE2, anti-SCGB1A1 and DAPI. c, Quantitative PCR for SARS-CoV-2 unspliced genomic RNA (gRNA, left) and spliced sgRNA (right) in apical-out distal lung organoids, $72 \mathrm{~h}$ post-infection (hpi), normalized to U3 snoRNA; $n=2$ independent experiments. d, Anti-dsRNA immunofluorescence of apical-out human distal lung organoids, either mock-infected or infected with
SARS-CoV-2, 48 hpi. e, Immunofluorescence for SARS-CoV-2 nucleocapsid protein (NP) in infected or mock-infected apical-out organoids at $96 \mathrm{hpi}$. f, Immunofluorescence of SARS-CoV-2-infected apical-out AT2 organoids with the indicated antibodies at $96 \mathrm{hpi}$. g, Immunofluorescence colocalization of SARS-CoV-2 NP and SCGB1A1 in apical-out distal lung basal organoids at $96 \mathrm{hpi}$. $\mathbf{h}$, Immunofluorescence showing cell-type specificity in SARS-CoV-2-infected apical-out organoids. Inf, SARS-CoV-2 infected cell. In $\mathbf{c - h}$, organoids were in suspension for 6-10 days (basal) or 3 days (AT2) before infection. Scale bars, $20 \mu \mathrm{m}$ except for $\mathbf{f}, \mathbf{h}(10 \mu \mathrm{m})$.
In basal organoids infected with SARS-CoV-2, double-stranded RNA (dsRNA) appeared by $48 \mathrm{~h}$ after infection (Fig. 4d) and SARS-CoV-2 nucleocapsid protein (NP) by $96 \mathrm{~h}$ (Fig. 4e). Approximately $10 \%$ of AT2 organoids displayed prominent SARS-CoV-2 NP expression in SFTPC ${ }^{+}$ cells; the remaining organoids were devoid of infection (Fig. 4f). Similarly, SARS-CoV-2 infected about $10 \%$ of basal organoids. In 2,621 total distal airway basal cells representing cultures from four individuals (Supplementary Table 5), SARS-CoV-2 infection was not detected in $\mathrm{KRT}^{+}$basal or AcTUB ${ }^{+}$ciliated cells (odds ratio $0, P<0.05$ ), in contrast to infection of upper airway ciliated cells by SARS-CoV-2 in 2D ALI culture ${ }^{29,30}$. However, SARS-CoV-2 NP and dsRNA immunofluorescence were primarily present in $\mathrm{SCGB1A1}^{+}$club cells (Fig. $4 \mathrm{~g}, \mathrm{~h}$ ) which were strongly associated with and accounted for $79 \%$ of NP- or dsRNA-positive cells (odds ratio 19.33, $P<0.0001$ ); $21 \%$ of infected cells lacked SCGB1A1 (Fig. 4g, h, Supplementary Table 5). Overall, these studies indicate that AT 2 cells were directly infected by SARS-CoV-2, and suggest that club cells are a distal lung target population.

\section{Discussion}

We have applied long-term human distal lung organoid cultures to progenitor discovery and modelling of infectious disease.
Our findings extend upon earlier short-term and feeder-dependent adult lung culture methods $s^{4,10,11}$ and present an alternative to techniques involving the differentiation of inducible pluripotent stem cells $\mathrm{s}^{31-34}$. Organoids contained two related subtypes of $\mathrm{KRT}^{+}$human distal lung basal cells-Basal 1 and Basal 2. Notably, the TNFRSF12A-expressing Basal 1 fraction possessed enriched clonogenic progenitor activity, establishing functional precedent for a proliferation-enriched basal cell subtype. Although TNFRSF12A is by no means exclusively present in basal cells, within the basal layer it often localizes to a postulated niche in airway furrow bases and tips ${ }^{23}$, extending recent notions that the lung epithelium shows spatial specialization ${ }^{35}$. It is possible that TNFRSF12A or analogous markers could distinguish basal cell progenitor subsets in other tissues. Our organoids also enable facile exploration of SARS-CoV-2 distal lung infection, which is relevant to COVID-19-associated pneumonia ${ }^{27}$, and implicate SCGB1A $1^{+}$club cells as a target whose infection could compromise protective lung glycosaminoglycans and precipitate a vicious infection cycle. We did not observe infection of ciliated cells, in contrast to 2D ALI lung studies ${ }^{29,30}$; this could require alternative culture conditions. SCGB1A1-negative populations were also infected and are under further investigation; for example, bronchial transient secretory cells express ACE2 and TMPRSS2 ${ }^{36}$. 
Overall, single-cell analysis of organoid cultures, as described here, may represent a general strategy for identifying and functionally validating candidate stem cells in slowly proliferating tissues. The culture of progenitors for all adult human distal lung epithelial lineages, including alveoli, should substantially enable the modelling of diseases such as neoplastic and interstitial lung conditions ${ }^{12}$ and allow tissue engineering and precision medicine applications. Finally, this organoid system should facilitate diverse investigations of pulmonary pathogens, including the SARS-CoV-2 distal lung infection that is associated with fulminant respiratory failure.

\section{Online content}

Any methods, additional references, Nature Research reporting summaries, source data, extended data, supplementary information, acknowledgements, peer review information; details of author contributions and competing interests; and statements of data and code availability are available at https://doi.org/10.1038/s41586-020-3014-1.

1. Hogan, B. \& Tata, P. R. Cellular organization and biology of the respiratory system Nat. Cell Biol. https://doi.org/10.1038/s41556-019-0357-7 (2019).

2. Rawlins, E. L. et al. The role of Scgb1a1 $1^{+}$Clara cells in the long-term maintenance and repair of lung airway, but not alveolar, epithelium. Cell Stem Cell 4, 525-534 (2009).

3. Kathiriya, J. J., Brumwell, A. N., Jackson, J. R., Tang, X. \& Chapman, H. A. Distinct airway epithelial stem cells hide among club cells but mobilize to promote alveolar regeneration. Cell Stem Cell 26, 346-358.e4 (2020).

4. Barkauskas, C. E. et al. Type 2 alveolar cells are stem cells in adult lung. J. Clin. Invest. 123, 3025-3036 (2013).

5. Desai, T. J., Brownfield, D. G. \& Krasnow, M. A. Alveolar progenitor and stem cells in lung development, renewal and cancer. Nature 507, 190-194 (2014).

6. Liu, Q. et al. Lung regeneration by multipotent stem cells residing at the bronchioalveolar-duct junction. Nat. Genet. 51, 728-738 (2019).

7. Vaughan, A. E. et al. Lineage-negative progenitors mobilize to regenerate lung epithelium after major injury. Nature 517, 621-625 (2015).

8. Zuo, W. et al. p63 ${ }^{+} \mathrm{Krt5}^{+}$distal airway stem cells are essential for lung regeneration. Nature 517, 616-620 (2015).

9. Juul, N. H., Stockman, C. A. \& Desai, T. J. Niche cells and signals that regulate lung alveolar stem cells in vivo. Cold Spring Harb. Perspect. Biol. https://doi.org/10.1101/ cshperspect.a035717 (2020).

10. Sucre, J. M. S. et al. Successful establishment of primary type II alveolar epithelium with 3D organotypic coculture. Am. J. Respir. Cell Mol. Biol. 59, 158-166 (2018).

11. Zacharias, W. J. et al. Regeneration of the lung alveolus by an evolutionarily conserved epithelial progenitor. Nature 555, 251-255 (2018)

12. Nikolić, M. Z. \& Rawlins, E. L. Lung organoids and their use to study cell-cell interaction. Curr. Pathobiol. Rep. 5, 223-231 (2017).

13. Evans, K. V. \& Lee, J. H. Alveolar wars: the rise of in vitro models to understand human lung alveolar maintenance, regeneration, and disease. Stem Cells Transl. Med. 9, 867-881 (2020)
14. Anchang, B. et al. Visualization and cellular hierarchy inference of single-cell data using SPADE. Nat. Protoc. 11, 1264-1279 (2016).

15. Cao, J. et al. The single-cell transcriptional landscape of mammalian organogenesis. Nature 566, 496-502 (2019).

16. Nabhan, A. N., Brownfield, D. G., Harbury, P. B., Krasnow, M. A. \& Desai, T. J. Single-cell Wnt signaling niches maintain stemness of alveolar type 2 cells. Science $359,1118-1123$ (2018).

17. Rock, J. R. et al. Basal cells as stem cells of the mouse trachea and human airway epithelium. Proc. Natl Acad. Sci. USA 106, 12771-12775 (2009)

18. Kajiji, S., Tamura, R. N. \& Quaranta, V. A novel integrin (alpha E beta 4) from human epithelial cells suggests a fourth family of integrin adhesion receptors. EMBO J. 8, 673-680 (1989).

19. Stange, D. E. et al. Differentiated Troy ${ }^{+}$chief cells act as reserve stem cells to generate all lineages of the stomach epithelium. Cell 155, 357-368 (2013).

20. Whitfield, M. L., George, L. K., Grant, G. D. \& Perou, C. M. Common markers of proliferation. Nat. Rev. Cancer 6, 99-106 (2006).

21. Rock, J. R. et al. Notch-dependent differentiation of adult airway basal stem cells. Cell Stem Cell 8, 639-648 (2011)

22. Pardo-Saganta, A. et al. Injury induces direct lineage segregation of functionally distinct airway basal stem/progenitor cell subpopulations. Cell Stem Cell 16, 184-197 (2015).

23. Quinton, P. M. Both ways at once: keeping small airways clean. Physiology (Bethesda) 32, 380-390 (2017)

24. Zhou, J. et al. Differentiated human airway organoids to assess infectivity of emerging influenza virus. Proc. Natl Acad. Sci. USA 115, 6822-6827 (2018).

25. Imai, M. \& Kawaoka, Y. The role of receptor binding specificity in interspecies transmission of influenza viruses. Curr. Opin. Virol. 2, 160-167 (2012).

26. Kumaki, Y., Day, C. W., Smee, D. F., Morrey, J. D. \& Barnard, D. L. In vitro and in vivo efficacy of fluorodeoxycytidine analogs against highly pathogenic avian influenza H5N1, seasonal, and pandemic H1N1 virus infections. Antiviral Res. 92, 329-340 (2011).

27. Zhu, N. et al. A novel coronavirus from patients with pneumonia in China, 2019. N. Engl. J. Med. 382, 727-733 (2020).

28. Co, J. Y. et al. Controlling epithelial polarity: a human enteroid model for host-pathogen interactions. Cell Rep. 26, 2509-2520.e4 (2019).

29. Lamers, M. M. et al. SARS-CoV-2 productively infects human gut enterocytes. Science 369, 50-54 (2020).

30. Hou, Y. J. et al. SARS-CoV-2 reverse genetics reveals a variable infection gradient in the respiratory tract. Cell 182, 429-446.e14 (2020).

31. Dye, B. R. et al. In vitro generation of human pluripotent stem cell derived lung organoids. elife 4, e05098 (2015)

32. Chen, Y. W. et al. A three-dimensional model of human lung development and disease from pluripotent stem cells. Nat. Cell Biol. 19, 542-549 (2017).

33. Jacob, A. et al. Differentiation of human pluripotent stem cells into functional lung alveolar epithelial cells. Cell Stem Cell 21, 472-488.e10 (2017).

34. Yamamoto, Y., Korogi, Y., Hirai, T. \& Gotoh, S. A method of generating alveolar organoids using human pluripotent stem cells. Methods Cell Biol. 159, 115-141 (2020).

35. Montoro, D. T. et al. A revised airway epithelial hierarchy includes CFTR-expressing ionocytes. Nature 560, 319-324 (2018)

36. Lukassen, S. et al. SARS-CoV-2 receptor ACE2 and TMPRSS2 are primarily expressed in bronchial transient secretory cells. EMBO J. 39, e105114 (2020).

Publisher's note Springer Nature remains neutral with regard to jurisdictional claims in published maps and institutional affiliations.

(c) The Author(s), under exclusive licence to Springer Nature Limited 2020 


\section{Methods}

Additional experimental details are in the Supplementary Methods. No statistical methods were used to predetermine sample size. The experiments were not randomized and the investigators were not blinded to allocation during experiments and outcome assessment.

\section{Human tissue procurement and processing}

All experiments performed in this work were approved by the Stanford University School of Medicine Institutional Review Board and performed under protocol no. 28908. Standard informed consent for research was obtained in writing from all patients who contributed to this study before tissue procurement and all experiments followed relevant guidelines and regulations. Peripheral lung tissue within $1 \mathrm{~cm}$ of the visceral pleura was obtained from discarded surgical tissue from lobectomies. For patients with suspected lung cancer, cases with clinical T4 (American Joint Cancer Committee 6th edition) disease (for example, features such as bronchial invasion or parenchymal satellite nodule/metastases) were deferred. Normal tissue was removed from the lung margin most anatomically distal to palpably well-defined lesions, or from uninvolved lobes in the case of pneumonectomies. Samples with tumours containing ill-defined margins were deferred. Tissue was either processed fresh or stored at $4{ }^{\circ} \mathrm{C}$ overnight and processed the following morning.

\section{Mixed distal lung organoid culture}

To isolate distal airway cells, lung parenchyma from $1 \mathrm{~cm}$ from the visceral pleura was mechanically dissociated with Castro scissors, washed and incubated with 5 units per ml porcine elastase (Worthington), 100 Kunitz units per ml DNase I (Worthington), and Normocin (InvivoGen), and resuspended in two tissue volumes of lung organoid medium, comprising advanced DMEM/F12 (Invitrogen) supplemented with $10 \mathrm{mM}$ nicotinamide, $N$-acetyl cysteine, $1 \times \mathrm{B} 27$ supplement minus vitamin A, recombinant human NOGGIN (100 $\mathrm{ng} \mathrm{ml}^{-1}, \mathrm{R} \& D$ Systems), recombinant human EGF (50 $\mathrm{ng} \mathrm{ml}^{-1}, \mathrm{R} \& D$ Systems), and TGF- $\beta$ inhibitor A83-01 (100 $\mathrm{nM}$, Tocris). This lung organoid medium was used for all experiments except for those shown in Extended Data Fig. 4f. The tissue was then agitated for $1 \mathrm{~h}$ at $37^{\circ} \mathrm{C}$ and the resultant cell suspension was filtered through $100-40-\mu \mathrm{m}$ cell strainers and subjected to ammonium chloride red blood cell lysis. The cell pellet was then washed and resuspended in 10 volumes of reduced growth factor Basement Membrane Extract II (Trevigen). Cells in matrix were then plated in 24-well plates in 50- $\mu$ l droplets, and warm medium was added after the droplets had solidified for $10 \mathrm{~min}$ at room temperature Medium was changed every 3-4 days and organoids were passaged every 3-4 weeks by dissociation with TrypLE. Passaging was based on ECM durability and integrity and estimated organoid confluency,judged by estimated organoid volume to volume of the ECM droplet. Distal lung organoids could be passaged for about 6 months with basal organoids initially exhibiting 6-7 doublings every 2 weeks. Alveolar organoids expanded more slowly with an initial rate of 3-4 doublings every 2 weeks but predominated over basal organoids after several months. Calculated from initial cell division rates, the upper limits of basal and alveolar expansion were $2^{19}\left(524,288\right.$-fold) and $2^{16}$ (65,536-fold), respectively. To rule out contamination by malignant cells, long-term cultures were systematically evaluated for the presence of dysplasia or carcinoma by a board-certified pathologist. In addition, five long-term organoid cultures (2-6 months) underwent targeted next generation sequencing to exclude pathogenic nucleotide variants (see below). Full details are provided in the Supplementary Methods.

\section{Tandem MACS stromal depletion and EPCAM purification of distal lung cells}

Distal lung was dissociated as above, and all incubation steps were carried out on ice. We incubated $10^{7}$ cells with Fc Block (Biolegend 422301) and diluted them 1:100 in FACS buffer ( 2 mM EDTA and 0.2\% fetal calf serum in $1 \times \mathrm{PBS}, \mathrm{pH} 7.4$ ), for $10 \mathrm{~min}$. The cells were then mixed with APC-conjugated anti-CD45 antibodies at $1 \mu \mathrm{g} \mathrm{ml}^{-1}$ in FACS buffer for $30 \mathrm{~min}$, washed, and subjected to two rounds of depletion with magnetic beads according to the manufacturer's protocol (Miltenyi: anti-human fibroblast 130-050-601, anti-CD31130-091-935, anti-APC130-090-855, LS column 130-042-401). Unlabelled cells were then centrifuged at $300 \mathrm{~g}$ and labelled with a cocktail of $1 \mu \mathrm{g} \mathrm{ml}^{-1}$ of PerCP-Cy5.5 anti-EPCAM antibody and Zombie Aqua viability stain (Biolegend 423101) diluted 1:400 from stock concentration in FACS buffer.

\section{Organoid cryopreservation and recovery}

For cryopreservation and recovery, ECM droplets were dissociated by pipetting in three volumes of PBS with 5 mM EDTA and then incubated on ice for $1 \mathrm{~h}$. Cells were pelleted at $300 \mathrm{~g}$ for $5 \mathrm{~min}$ and resuspended in freezing medium (fetal calf serum (Gibco), $10 \% \mathrm{v} / \mathrm{v}$ DMSO), placed into cryovials and then into Mr. Frosty (Thermo Fisher) containers and stored at $-80^{\circ} \mathrm{C}$ overnight, followed by transfer to liquid nitrogen vapour phase for long-term storage. Organoids were recovered by quick thaw in a $37^{\circ} \mathrm{C}$ water bath followed by washing in organoid medium and plating in ECM with organoid medium plus $10 \mu \mathrm{M} \mathrm{ROCK}$ inhibitor Y-27632 (Tocris).

\section{Screening exogenous growth factors in organoid culture}

Distal airway cells were isolated and plated as above with the following exceptions: advanced DMEM/F12 was used instead of organoid medium during elastase digestion of lung tissue, and cells were serially diluted and filtered through a $40-\mu \mathrm{m}$ cell strainer and counted with a haemocytometer. One thousand viable epithelial cells (by Trypan blue exclusion, size, and morphology) per $\mu \mathrm{l}$ ECM were plated per $5 \mu \mathrm{l}$ Matrigel droplet per well. Base medium consisted of organoid medium lacking A83-01, EGF, NOGGIN, WNT3A or RSPO1. EGF (final $50 \mathrm{ng} \mathrm{ml}^{-1}, \mathrm{R} \& \mathrm{D}$ ), NOGGIN (final $100 \mathrm{ng} \mathrm{ml}^{-1}, \mathrm{R} \& \mathrm{D}$ ), WNT3A (final $100 \mathrm{ng} \mathrm{ml}^{-1}, \mathrm{R} \& \mathrm{D}$ ), RSPO1 (final $500 \mathrm{ng} \mathrm{ml}^{-1}$, Peprotech) or the PORCUPINE inhibitor C59 (final $1 \mu \mathrm{M}$, Biogems) were added singly or in combination to base medium. Images were obtained ten days after primary plating with an inverted light microscope at $5 \times$ magnification. Each condition was plated in quadruplicate and organoid formation was quantified using the analyse particle (threshold, $490^{2}$ pixels) plugin in ImageJ (see Supplementary Methods).

\section{Single-cell RNA-seq of unfractionated organoid cultures}

Lung organoid cultures from separate individuals were dissociated 4 weeks after primary plating and subjected to droplet-based scRNA-seq with the 10x Genomics Single Cell 3' platform with a 5-nucleotide UMI, according to the manufacturer's protocol. Cell capture, library preparation, and sequencing were performed as previously described ${ }^{37}$. For scRNA-seq analysis in Fig. 1k, a modified Kruskal-Wallis rank sum test was performed to determine the significance of differential marker gene expression for AT2 (SFTPC), basal (KRT5), and club (SCGB1A1) cells, with all $P<0.001$. Principal component analysis, t-SNE, unsupervised graph-based clustering, statistical testing, and the pseudotime trajectory for all scRNA-seq analyses are described in Supplementary Methods and Supplementary Data 1.

\section{Single-cell RNA-seq of purified AT2 organoid cultures}

LysoTracker ${ }^{+}$AT 2 cells $^{38}$ from unfractionated organoids were purified by FACS and cultured for two months with one passage. These were dissociated and subjected to droplet-based scRNA-seq with the 10x Genomics Chromium Single Cell 3' platform v2 according to the manufacturer's protocol. The library was sequenced using paired-end sequencing (26bp read 1 and 98 bp read 2 ) with a single sample index ( $8 \mathrm{bp}$ ) on an Illumina NextSeq 500. Data preprocessing and principal component analysis were carried out with CellRanger v1.2. Subsequent analysis is described in Supplementary Methods and Supplementary Data 1. 


\section{Electron microscopy}

Organoid cultures were fixed in ECM with $2.5 \%$ glutaraldehyde in $0.1 \mathrm{M}$ cacodylate buffer ( $\mathrm{pH} 7.4$ ), dehydrated, embedded in epoxy resin and visualized with a JEOL (model JEM1400) transmission-electron microscope with a LaB6 emitter at $120 \mathrm{kV}$.

\section{Histology and immunocytochemistry}

Organoids were fixed with $2 \%$ paraformaldehyde at $4{ }^{\circ} \mathrm{C}$ overnight, paraffin embedded and sectioned $(10-20 \mu \mathrm{m})$ as previously described ${ }^{37}$. Sections were deparaffinized and stained with H\&E for histological analysis. Antibodies used for immunocytochemistry staining following standard staining protocols $\mathrm{s}^{39}$ are listed in Supplementary Methods and images were acquired on a Leica-SP8 confocal microscope.

\section{RNA fluorescent in situ hybridization}

RNA in situ hybridization was performed as described ${ }^{40}$ and probe sequences are provided in Supplementary Methods.

\section{Whole-mount organoid confocal immunofluorescence microscopy}

Intact, uninfected organoids were fixed in $2 \%$ paraformaldehyde in $100 \mathrm{mM}$ phosphate buffer ( $\mathrm{pH} 7.4)$ (4\% paraformaldehyde for infected organoids) for $1 \mathrm{~h}$ at room temperature, washed with PBS with $100 \mathrm{mM}$ glycine, permeabilized in $0.5 \%$ Triton X-100 in PBS for $1 \mathrm{~h}$, then incubated in staining buffer (4\% BSA, 0.05\% Tween-20 in PBS pH 7.4, 10\% goat/donkey serum) for an additional hour, followed by incubation with primary antibody for $24 \mathrm{~h}$ at room temperature in staining buffer. Whole mounts were then washed with PBS-T and incubated with fluorescent secondary antibodies, phalloidin and DAPI, for $4 \mathrm{~h}$ at room temperature in staining buffer. Following additional washes, whole mounts were submerged in mounting medium (Vectashield, Vector Laboratories) and mounted on chambered coverslips for imaging in four channels using Zeiss LSM 700 or 900 confocal microscopes. 3D rendering of confocal image stacks was performed using Volocity Image Analysis software (Quorum Technologies Inc., Guelph, Ontario). For Fig. 4h, which required five colours, cilia were distinguished by staining with two fluorescent secondary antibodies and merging the colocalized voxels into a pseudocoloured channel using Volocity software. Lectin staining (FITC-Sambuca Nigrin, Vector Labs FL-1301; Biotin-Maackia Amurensis, Vector Labs FL-1301) was carried out according to the manufacturer's protocol after fixation of organoids with $0.1 \%$ paraformaldehyde in PBS for $1 \mathrm{~h}$ at room temperature followed by blocking with avidin/biotin (Vector Labs SP-2001). Biotin-Maackia Amurensis lectin was labelled with streptavidin-PE conjugate (Thermo Fisher SA10041) and after washing lectin staining was imaged in a Keyence BZ-X700.

\section{Next generation sequencing of organoid cultures}

Ten organoid cultures were sequenced using a commercial targeted resequencing assay with end-to-end coverage of 131 cancer genes and companion software (TOMA COMPASS Tumour Mutational Profiling System, Foster City, CA) to determine the presence of oncogenic mutations in long-term organoid cultures. Libraries were sequenced on an Illumina NextSeq 500. Nonsynonymous variants are listed in Supplementary Table 6. Variant call files are provided in Supplementary Data 2.

\section{Density sedimentation of basal cells}

Organoid cultures within 2-3 weeks of primary plating were dissociated with $1 \mathrm{U} \mathrm{ml}^{-1}$ neutral protease (Worthington, Cat LS02100) and $100 \mathrm{KU}$ DNase I in lung organoid medium. Basal organoids were then collected by gravity sedimentation and the supernatant was either aspirated or collected for downstream use. Basal organoids were then further fractionated on a custom Ficoll-Paque gradient (4 vol Ficoll-Paque to $1 \mathrm{vol}$ PBS) and centrifuged at $300 \mathrm{~g}$ for $10 \mathrm{~min}$ at room temperature. Supernatant was aspirated and the organoid pellet was resuspended in $10 \mathrm{ml}$ PBS in a 15-ml conical tube, collected by gravity sedimentation, and plated into ECM as described above.

\section{FACS isolation and culture of AT2 cells}

Organoids were dissociated with TrypLE followed by neutralization with $10 \%$ volume fetal calf serum, subjected to DNase at $100 \mathrm{KU} \mathrm{ml}^{-1}$, washed with lung organoid medium and then incubated with 100 cell pellet volumes of lung organoid medium with $10 \mathrm{nM}$ LysoTracker Red DND-99 (Thermo Fisher L7528) at $37^{\circ} \mathrm{C}$ for $30 \mathrm{~min}$. Cells were then washed and resuspended in FACS buffer as described above, incubated with Fc block, and then incubated on ice with labelling cocktail consisting of $1 \mu \mathrm{g} \mathrm{ml}^{-1}$ of PerCP-Cy5.5 anti-EPCAM antibody and Zombie Aqua viability stain (Biolegend 423101) diluted 1:400 from stock concentration in FACS buffer. EPCAM ${ }^{\text {hi }}$ and LysoTracker ${ }^{\text {hi }}$ cells were sorted into lung organoid medium with $10 \mu \mathrm{M}$ Y-27632 (Tocris 1254) and cultured in ECM and lung organoid medium with Y-27632 for $24 \mathrm{~h}$, followed by lung organoid medium without Y-27632. Pure AT2 organoid growth was enhanced by the addition of 1:1 vol:vol serum-containing L-cell conditioned medium (L-WRN CM) containing WNT3A, R-SPONDIN3 and NOGGIN and supplemented with recombinant $E^{4} F^{41}$. The full gating strategy is provided in Supplementary Fig. 1. All FACS antibodies were purchased from Biolegend. Qualitatively identical results could also be obtained with anti-HTII-280 (AT2 marker, Terrace Biotech) FACS purification in lieu of LysoTracker. AT2 organoid cells were transdifferentiated into AT1 cells by TrypLE dissociation from ECM and seeding onto chambered glass coverslips followed by culturing with advanced DMEM/F12 and 5\% fetal calf serum ${ }^{42}$.

\section{Colour mixing studies with lentivirally transduced GFP and mCherry}

FACS EPCAM $^{+}$stroma-depleted organoids at D14 were infected with lentivirus at an estimated MOI of 0.9 as described previously ${ }^{43}$ with third-generation lentiviral vectors (PGK-GFP T2A Puro, SBI cat. no. CD550A-1; mCherry modified from pLentiCRISPRv1 (Addgene no. 49545) to incorporate an EF-1a-mCherry P2A Puro cassette, a gift from Paul Rack). Ninety-six hours after infection, organoids were treated with puromycin at a concentration of $600 \mathrm{ng} \mathrm{ml}^{-1}$ for $48 \mathrm{~h}$ to select for transduced cells. Two weeks after selection, GFP-expressing organoids or mCherry-expressing organoids were dissociated into single cells and mixed in a 1:1 ratio and scored as monochromatic or mixed after 28 days of each passage. The same approach was employed for purified AT2 and basal cultures after respective isolation strategies from an initial FACS-purified, $\mathrm{EPCAM}^{+}$, stroma-depleted organoid starter culture.

\section{Flow cytometry analysis of resident basal cells from adult human lung}

Adult human lung tissue was procured and dissociated as above but cells were labelled with Zombie Aqua live:dead stain as above, washed with FACS buffer, and then fixed in $2 \%$ PFA in PBS overnight at $4{ }^{\circ} \mathrm{C}$. Cells were then stained using the whole-mount procedure as described above with the omission of PBS-glycine washing. Fixed and permeabilized cells were then incubated with 1:400 dilution of Alexa Fluor 647 conjugated mouse anti-human cytokeratin 5 antibody (Abcam) for $24 \mathrm{~h}$ at $4^{\circ} \mathrm{C}$ in permeabilization buffer. Cells were then washed with FACS buffer and labelled with PE conjugated mouse anti-human TNFRSF12A antibody (clone ITEM-4, Biolegend) for $30 \mathrm{~min}$ on ice, followed by washing and analysis on a BD Aria Fusion instrument. The full gating strategy and qPCR validation of the ITEM-4 antibody are detailed in Supplementary Fig. 1.

\section{FACS isolation of TNFRSF12 $A^{\text {hi }}$ and TNFRSF12 $A^{\text {neg }}$ basal cells}

Single-cell suspensions from either fresh human distal lung or primary organoid culture at approximately 4 weeks of culture were dissociated as above, treated with Fc Block (BioLegend), and incubated in FACS buffer with Zombie Aqua 1:400,1 $\mathrm{g} \mathrm{m} \mathrm{ml}^{-1}$ PerCP-Cy5.5 anti-human 
EpCAM (CD326), $1 \mu \mathrm{g} \mathrm{ml}^{-1} \mathrm{APC}$ anti-human ITGA6 (CD49f), $2 \mu \mathrm{g} \mathrm{ml}^{-1} \mathrm{FITC}$ anti-human ITGB4 (CD104), and $1 \mu \mathrm{g} \mathrm{ml}^{-1} \mathrm{PE}$ anti-human TNFRSF12A (CD266). Thirty minutes after labelling, the cells were washed twice with FACS buffer and sorted for EPCAM ${ }^{\text {hi }}$, ITGA6/ITGB4 ${ }^{\text {hi }}$, TNFRSF12A ${ }^{\text {hi }}$ and TNFRSF12 $\mathrm{A}^{\text {neg }}$. The full gating strategy is provided in Supplementary Fig. 1. More than 5,000 cells were sorted into Eppendorf tubes with lung organoid medium and $10 \mu \mathrm{M}$ ROCK inhibitor Y-27632. All FACS antibodies were purchased from Biolegend.

\section{Culture of TNFRSF12A ${ }^{\text {hi }}$ and TNFRSF12 $A^{\text {neg }}$ basal cells}

Cells were seeded in ECM and submerged in lung organoid medium with $10 \mu \mathrm{M}$ ROCK inhibitor Y-27632. The seeding density for cells FACS-isolated from organoid culture was 1,000 cells per well at a density of 100 cells per $\mu \mathrm{lECM}$. The seeding density for cells FACS-isolated from fresh human distal lung was 3,000 cells per well at a density of 300 cells per $\mu \mathrm{IECM}$. After $24 \mathrm{~h}$, the medium was changed to remove ROCK inhibitor, thereafter it was changed every $72 \mathrm{~h}$. Organoid formation was manually quantified 14 days after plating by two independent observers.

\section{H1N1 organoid influenza assay}

Unfractionated cultures containing AT2, basal, and club cell types at 2-3 weeks were infected in triplicate with H1N1 strain PR8 modified to express GFP upon viral replication ${ }^{44}$ after $24 \mathrm{~h}$ of pretreatment with antiviral compounds. ECM was dispersed by addition of 5 mMEDTA in PBS, followed by washing and inoculation with PR8-H1N1-GFP reporter virus at an estimated $\mathrm{MOI}$ of 1 in medium containing either vehicle or antivirals. After $12 \mathrm{~h}$ (one influenza infection cycle), intact organoid GFP expression was either visualized by fluorescence microscopy with a Keyence BZ-X700 automated microscope, or dissociated to single cells and fixed with $0.1 \%$ PFA in PBS followed by FACS quantification of $\mathrm{GFP}^{+}$cells (gating strategy is provided in Supplementary Fig. 1). Antiviral dose response curves were generated using four-parameter nonlinear regression curve fitting with GraphPad Prism 7 (GraphPad Software, San Diego, CA). H1N1 tropism was assessed in a manner similar to above with the exception that Ficoll-sedimented basal cell fraction versus non-basal fractions were dissociated into single cells, counted, and infected with an estimated MOI of 1 in organoid medium for $1 \mathrm{~h}$ at $37^{\circ} \mathrm{C}$, followed by washing and reseeding into ECM, culturing for $16 \mathrm{~h}$, and then being subjected to dissociation and FACS as above.

\section{Suspension culture to generate apical-out polarity in lung organoids}

Lung organoids grown embedded in $50 \mu \mathrm{l} \mathrm{ECM} \mathrm{droplets} \mathrm{were} \mathrm{trans-}$ ferred to suspension culture as described ${ }^{28}$ with modifications. In brief, ECM-embedded organoids were dislodged gently by pipetting using sterile LoBind tips (Eppendorf 22493008) and placed in $15 \mathrm{ml}$ LoBind conical tubes (Eppendorf 30122216) containing ice-cold 5 mMEDTA in PBS. Five millilitres of EDTA solution was used per ECM droplet (3 ECM droplets per $15-\mathrm{ml}$ conical tube) rotating for $1 \mathrm{~h}$ at $4{ }^{\circ} \mathrm{C}$ on a rotating platform. Organoids were centrifuged at $200 \mathrm{~g}$ for $3 \mathrm{~min}$ at $4{ }^{\circ} \mathrm{C}$ and the supernatant was removed. The pellet was resuspended in growth medium in ultra-low attachment six-well tissue culture plates (Corning Costar 3471). Suspended organoids were incubated at $37^{\circ} \mathrm{C}$ with $5 \%$ $\mathrm{CO}_{2}$ for different times (range 0-30 days) to characterize apical-out polarity, ciliogenesis and differentiation, and to prepare apical-out organoids for infection experiments with SARS-CoV-2.

\section{SARS-CoV2 infection of human distal lung organoids}

VeroE6 cells were obtained from ATCC as mycoplasma-free stocks and maintained in supplemented DMEM with $10 \%$ FBS. SARS-CoV-2 (USA-WA1/2020) was passaged in VeroE6 cells in DMEM with $2 \%$ FBS. Titres were determined by plaque assay on VeroE6 cells using Avicel (FMC Biopolymer) and crystal violet (Sigma), the viral genome sequence was verified, and all infections were performed with passage
3 virus. Organoids were counted and passaged into suspension medium for 6-8 days and then resuspended in virus medium or an equal volume of mock medium at an $\mathrm{MOI}$ of 1 relative to total organoid cells in the sample, and then incubated at $37^{\circ} \mathrm{C}$ under $5 \% \mathrm{CO}_{2}$ for $2 \mathrm{~h}$. Organoids were then plated in suspension in lung organoid medium (apical-out organoids). At the indicated time points, apical-out organoids were washed with lung organoid medium and PBS and resuspended in TRIzol LS (Thermo Fisher), freshly-made $4 \%$ PFA in PBS, or $250 \mu$ l lung organoid medium. Cells resuspended in lung organoid medium were lysed by freezing at $-80^{\circ} \mathrm{C}$. Culture supernatants were preserved in TRIzol LS or added directly to plaque assay monolayers. All SARS-CoV-2 work was performed in a class II biosafety cabinet under BSL3 conditions at Stanford University.

\section{qPCR analysis of SARS-CoV-2 RNA}

RNA from SARS-CoV-2-infected organoids was extracted by adding $750 \mu \mathrm{l} \mathrm{TRIzol}$ (Thermo Fisher Scientific), incubating at $55^{\circ} \mathrm{C}$ for $5 \mathrm{~min}$ and then adding $150 \mu \mathrm{l}$ chloroform. After mixing each sample by vortexing for $7 \mathrm{~s}$, the samples were incubated at $25^{\circ} \mathrm{C}$ for $5 \mathrm{~min}$ and then centrifuged at $12,000 \mathrm{rpm}$ for $15 \mathrm{~min}$ at $4{ }^{\circ} \mathrm{C}$. The aqueous layer was carefully removed from each sample, mixed with two volumes of $100 \%$ ethanol and purified using an RNA Clean \& Concentrator-25 kit (Zymo Research) as per the manufacturer's instructions. All RNA samples were treated with DNase (Turbo DNA-free kit, Thermo Fisher Scientific). The Brilliant II SYBR Green QRT-PCR 1-Step Master Mix (VWR) was used to convert RNA into cDNA and to amplify specific RNA regions on the CFX96 Touch real-time PCR detection system (Bio-Rad). The reverse transcription reaction was performed for $30 \mathrm{~min}$ at $50^{\circ} \mathrm{C}$, 10 min at $95^{\circ} \mathrm{C}$, followed by two-step qPCR with $95^{\circ} \mathrm{C}$ for $10 \mathrm{~s}$ and $55^{\circ} \mathrm{C}$ for $30 \mathrm{~s}$, for a total of 40 cycles. Two primer sets were used to amplify either non-spliced SARS-CoV-2 genomic RNA (gRNA) spanning nucleotide positions 14221-14306, or spliced SARS-CoV-2 sgRNA ${ }^{30}$. Primer sequences are in Supplementary Table 7.

\section{TNFRSF12A immunostaining of intact distal lung}

Optimal staining of human distal lung tissue was achieved from specimens fixed within 30 min of primary surgical resections in $4 \%$ paraformaldehyde in PBS. Specimens were incubated in fixative overnight at $4{ }^{\circ} \mathrm{C}$, transferred to $30 \%$ sucrose, and embedded into OCT. Frozen sections were cut at $10 \mu \mathrm{m}$, subjected to citrate-based antigen retrieval (Vector Labs) at $70{ }^{\circ} \mathrm{C}$ for $30 \mathrm{~min}$, and then blocked for $1 \mathrm{~h}$ with $10 \%$ goat serum in IF wash buffer as described above. Mouse anti-TNFRSF12A (clone ITEM-4, Biolegend) was used for Fig. 3a and polyclonal rabbit anti-TNFRSF12A (ThermoFisher PA5-20275) was used for Fig. 3b, fand Extended Data Fig. $8^{45}$.

\section{Live-imaging and confocal microscopy of immobilized apical-out lung organoids}

Live organoids were held between two coverslips in a viewing chamber (Lab-Tek II two-chambered coverglass) and filmed using a Nikon TE2000E microscope using differential interference contrast (DIC) microscopy with a $63 \times$ objective. Samples were kept at $37^{\circ} \mathrm{C}$ with $5 \%$ $\mathrm{CO}_{2}$ during imaging. Digital videos were collected by a Hamamatsu high-resolution ORCA-285 digital camera and rendered using OpenLab 5.5.2 software (Improvision). After recording, samples were fixed and stained without removal from the chambers and transferred to the confocal microscope for immunofluorescence microscopy.

\section{Statistics and reproducibility}

Unless stated otherwise, all data are representative of at least two independent experiments with each independent experiment carried out using an organoid culture derived from one individual. Box plot bounds span first through third quartiles, horizontal lines represent median values, and whiskers represent data range minima or maxima or, in the case of outliers, 1.5 times the interquartile range with outliers 
represented by data points. $t$-tests were two-tailed and $P$ values are denoted as ${ }^{*} P<0.05,{ }^{* *} P \leq 0.01$, and ${ }^{* * *} P \leq 0.001$. Full details are provided in Supplementary Methods.

\section{Reporting summary}

Further information on research design is available in the Nature Research Reporting Summary linked to this paper.

\section{Data availability}

scRNA-seq data sets have been deposited in Gene Expression Omnibus with the accession code GSE106850. Source data are provided with this paper.

\section{Code availability}

Scripts to perform analyses of scRNA-seq data are provided with this paper. Custom code is available on GitHub (https://github.com/ ameen-salahudeen/lung_organoid).

37. Yan, K. S. et al. Non-equivalence of Wnt and R-spondin ligands during Lgr $5^{+}$intestinal stem-cell self-renewal. Nature 545, 238-242 (2017)

38. Van der Velden, J. L., Bertoncello, I. \& McQualter, J. L. LysoTracker is a marker of differentiated alveolar type II cells. Respir. Res. 14, 123 (2013).

39. Chang, J. et al. Gpr124 is essential for blood-brain barrier integrity in central nervous system disease. Nat. Med. 23, 450-460 (2017).

40. Nagendran, M., Riordan, D. P., Harbury, P. B. \& Desai, T. J. Automated cell-type classification in intact tissues by single-cell molecular profiling. eLife 7, e30510 (2018)

41. Neal, J. T. et al. Organoid modeling of the tumor immune microenvironment. Cell 175, 1972-1988.e16 (2018)

42. Dobbs, L. G., Williams, M. C. \& Brandt, A. E. Changes in biochemical characteristics and pattern of lectin binding of alveolar type II cells with time in culture. Biochim. Biophys. Acta 846, 155-166 (1985).

43. Van Lidth de Jeude, J. F., Vermeulen, J. L., Montenegro-Miranda, P. S., Van den Brink, G. R. \& Heijmans, J. A protocol for lentiviral transduction and downstream analysis of intestinal organoids. J. Vis. Exp. 98, e52531 (2015).

44. Manicassamy, B. et al. Analysis of in vivo dynamics of influenza virus infection in mice using a GFP reporter virus. Proc. Natl Acad. Sci. USA 107, 11531-11536 (2010).

45. Karaca, G. et al. TWEAK/Fn14 signaling is required for liver regeneration after partial hepatectomy in mice. PLOS ONE 9, e83987 (2014).
Acknowledgements We thank members of the Kuo and Desai laboratories for discussions: the Stanford Tissue Bank, J. Shrager, M. Berry and W. Trope for tissue acquisition; S. Plevritis for trajectory analysis; and the Stanford Stem Cell FACS Facility, P. Chu, A. McCormick, D. Mendoza, F. de la Vega and J. Zengel for technical expertise. SARS-Related Coronavirus 2, Isolate USA-WA1/2020, NR-52281 was deposited by the CDC and obtained through BEI Resources, NIAID, NIH. Fellowships supporting authors are as follows: A.A.S.: A.P. Giannini, ECOG-ACRIN, P. Carbone, Stanford Cancer Institute; S.S.C.: Stanford Medical Scientist Training Program grant T32 GM007365-44; J.Z.: Stanford Graduate Fellowship.; S.M.d.I.O.: CIRM Bridges. Funding support is as follows: A.R.: NIH grant T32 AI007502-23; R.A.F. Damon Runyon Cancer Research Foundation (DRG-2286-17); V.v.U.: Netherlands Organization for Scientific Research Rubicon grant (452181214); C.S. and J.Z.: NSF DMS 1712800 and the Stanford Discovery Innovation Fund: K.C.G. and M.M.D.: Howard Hughes Medical Institute; C.A.B.: Burroughs Wellcome Fund Investigators in the Pathogenesis of Infectious Disease Grant 1016687. This work was also supported by CIRM DISC2-09637 to C.J.K. and T.J.D.: Bill and Melinda Gates Foundation OPP1113682 to C.J.K., M.R.A., and C.A.B.; Novo Nordisk Foundation Challenge Grant to M.R.A and M.M.-C.; Mathers Foundation Covid Fund to K.C.G.; and NIH grants K08DEO27730 to A.A.S., U19AIO57229 to M.M.D., R56Al111460 to J.S.G. 5R01HI14254902 to T. D. DK11572802 to C.J.K. and K.C.G. R01Al157155 to R.S.B., and U19Al116484, U01DK085527, U01CA217851, U01CA176299 and U01DEO25188 to C.J.K.. C.A.B is the Tashia and John Morgridge Faculty Scholar and Chan Zuckerberg Biohub Investigator. T.J.D. is the Woods Family Endowed Faculty Scholar in Pediatric Translational Medicine. C.J.K. is the Maureen Lyles D'Ambrogio Professor of Medicine.

Author contributions A.A.S. and S.S.C. conceived, designed, and performed experiments, analysed data, and wrote the manuscript. A.R. designed and performed SARS-CoV-2 infections. J.Z., V.v.U. and C.S. designed and interpreted single-cell RNA-seq studies. R.A.F. performed qRT-PCR. M.M.-C., S.M.d.I.O., A.J.M.S., T.U., D.J.H., J.J. and A.B. performed organoid culture and analysis. L.E.W. and M.M.D. designed FACS panels. V.L. and K.C.G. contributed the DLL4 E12 mutant. B.A. performed SPADE analysis. K.N. and J.S.G. designed and executed influenza studies. G.X.Y.Z., J.M.T., P.B., S.B.Z. and T.S.M. designed and executed single-cell RNA-seq experiments. C.E.E. and R.S.B. advised on SARS-CoV-2 studies. M.N. and P.B.H. provided in situ hybridization protocols. M.R.A. C.A.B., T.J.D. and C.J.K. conceived and designed experiments, analysed data and wrote the manuscript.

Competing interests C.J.K., A.A.S., S.S.C., C.A.B., A.R., M.R.A., M.M.-C., S.M.d.I.O. and T.U. are listed as inventors on provisional patent $63 / 053,079$ describing the methods in this paper. C.J.K. is a founder of Surrozen Inc. All other authors declare no competing interests.

\section{Additional information}

Supplementary information is available for this paper at https://doi.org/10.1038/s41586-020 3014-1.

Correspondence and requests for materials should be addressed to C.A.B., T.J.D. or C.J.K. Peer review information Nature thanks the anonymous reviewers for their contribution to the peer review of this work.

Reprints and permissions information is available at http://www.nature.com/reprints. 
a

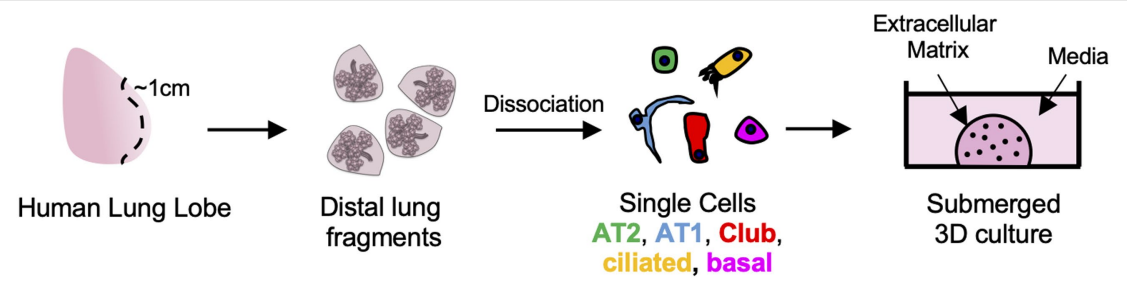

b

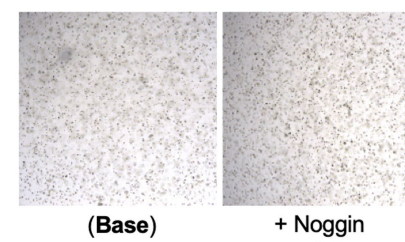

(N)

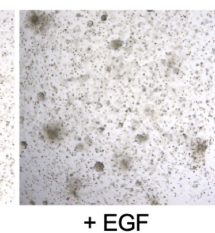

(E)

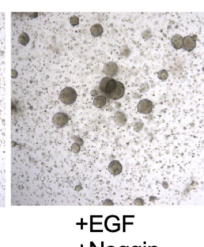

+Noggin

(EN)

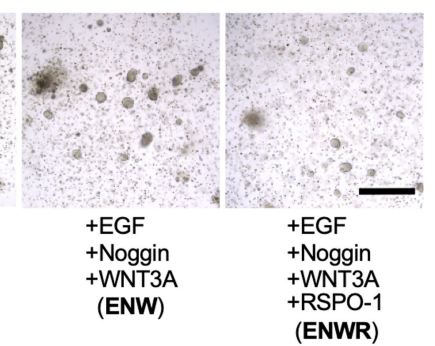

(ENWR)

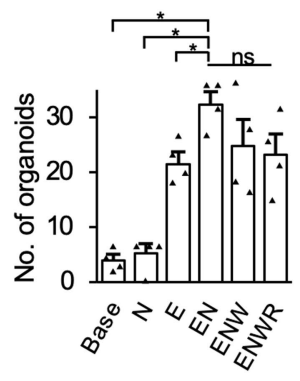

C

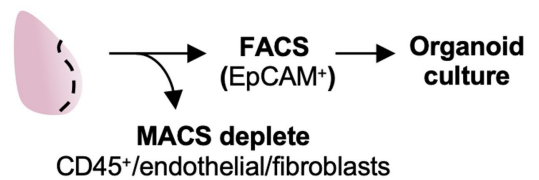

CD45+/endothelial/fibroblasts
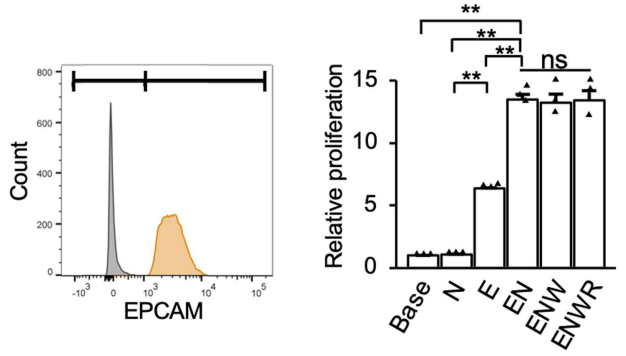

$\mathbf{e}$

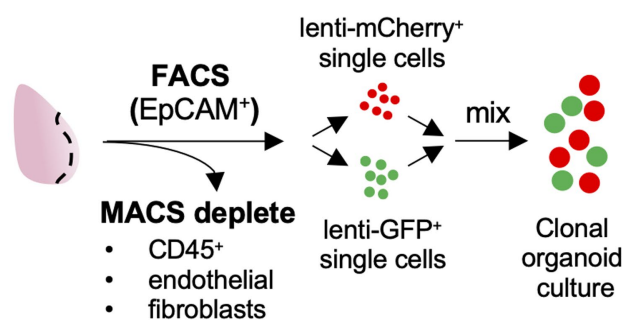

d day

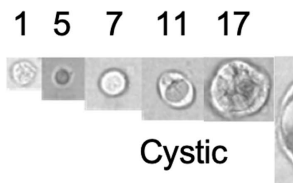

23

28

32

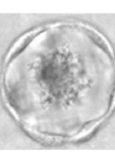

day
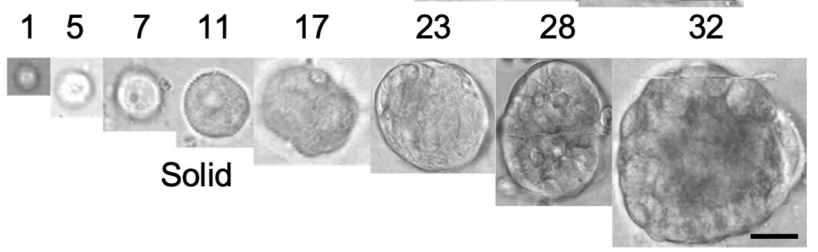

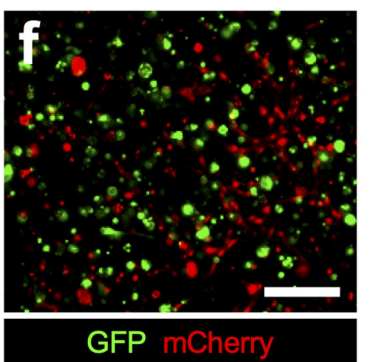

\begin{tabular}{|l|c|c|c|}
\hline & Red & Green & Chimera \\
\hline Lung 1 & 110 & 107 & 1 \\
\hline Lung 1 P1 & 93 & 100 & 0 \\
\hline Lung 2 & 98 & 113 & 0 \\
\hline Lung 2 P1 & 110 & 102 & 1 \\
\hline
\end{tabular}

Extended Data Fig. 1 | Optimization of human distal lung organoid culture. a, Schematic of culture initiation from human distal lung. b, Brightfield microscopy evaluation of required exogenous growth factors and automated organoid quantitation after day 10 of chemically defined organoid culture with specified recombinant growth factors, $N=$ Noggin, $\mathrm{E}=\mathrm{EGF}, \mathrm{W}=\mathrm{WNT} 3 \mathrm{~A}$, $\mathrm{R}=\mathrm{RSPO1}, n=4$ per condition, data are mean \pm s.e.m. ${ }^{*}=P<0.05$, two-tailed student's $t$-test, scale bar $=500 \mu \mathrm{m}$. c, Top, purification schema to isolate epithelial cells from distal human lung involving negative MACS bead depletion of $\mathrm{CD} 45^{+}$haematopoietic cells, endothelial cells and fibroblasts, followed by positive FACS selection for $\mathrm{EPCAM}^{+}$epithelium. Bottom left, representative FACS demonstrating $>99.9 \% \mathrm{EPCAM}^{+}$purity (orange) upon re-analysis versus unstained controls (grey). Bottom right, proliferation of
$\mathrm{EPCAM}^{+}$cells purified from distal lung cultures after day 10 of organoid culture with specified growth factors, $N=$ Noggin, $\mathrm{E}=\mathrm{EGF}, \mathrm{W}=\mathrm{WNT} 3 \mathrm{~A}, \mathrm{R}=\mathrm{RSPO}, n=3$ per condition, data are mean \pm s.e.m., ${ }^{* *}=P<0.01$, two-tailed student's $t$-test. d, Time lapse transmission confocal images of solid and cystic organoids originating from single dissociated human distal lung cells, scale bar $=100 \mu \mathrm{m}$. e-g, Clonality mixing studies. e, Schema of mixing studies of lentivirusGFP- and lentivirus-mCherry-expressing cells to determine clonality. f, Representative live fluorescent imaging of resultant green and red organoids from (e), scale bar $=500 \mu \mathrm{m} . \mathrm{g}$, Quantitation of red, green, or chimaeric, distal lung organoid cultures from two individuals $(1,2)$ after initial and serial passaging $(\mathrm{P} 1=$ passage 1$)$. 

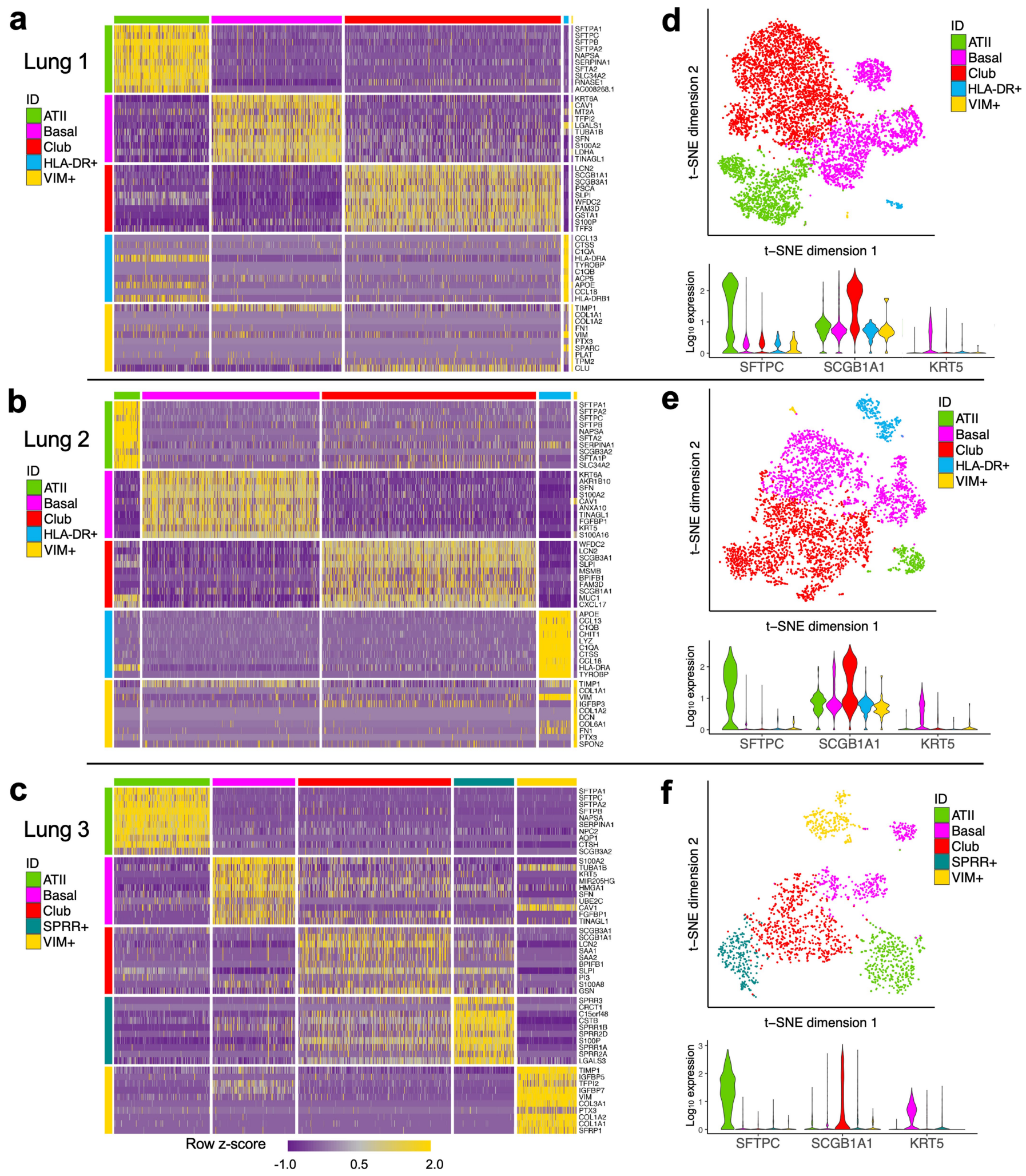

Extended Data Fig. 2 | scRNA-seq of human distal lung organoids cultured from three individuals reveals reproducible basal, club, and AT2 populations. a-c, Unsupervised clustering of total cell populations

remainder being either fibroblasts $\left(V I M^{+}\right)$or mononuclear cells (HLA-DR+, likely alveolar macrophages). $\mathbf{d}-\mathbf{f}, \mathrm{t}$-SNE visualization and violin plots for marker genes corresponding to each population. Note, a unique population enriched for SPRR genes, which have been described to mark squamous metaplasia, were exclusively found in the organoid culture of Lung 3, derived from an individual who was an active smoker. to basal (KRT5/6), club (SCGB1A1), and AT2 (SFTPC) cells. The epithelial fraction from these cultures ranged from approximately $90-99 \%$ of all cells with the 


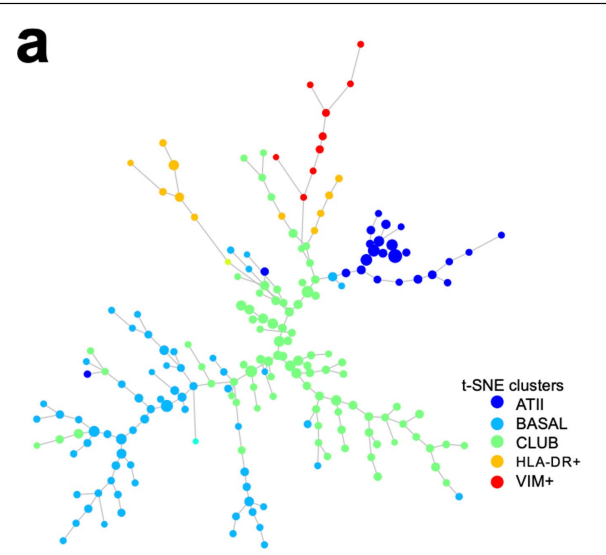

d

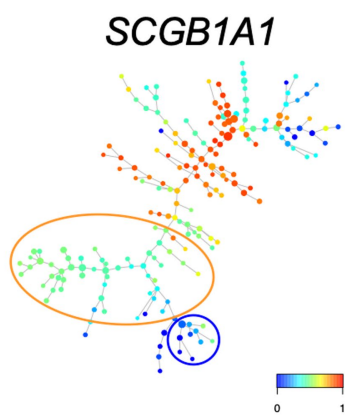

b

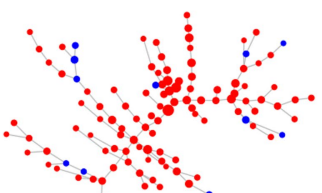

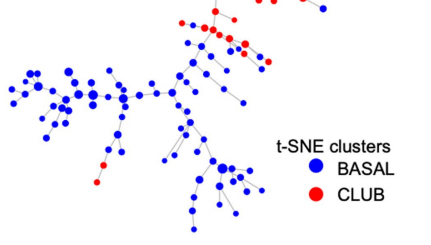

C

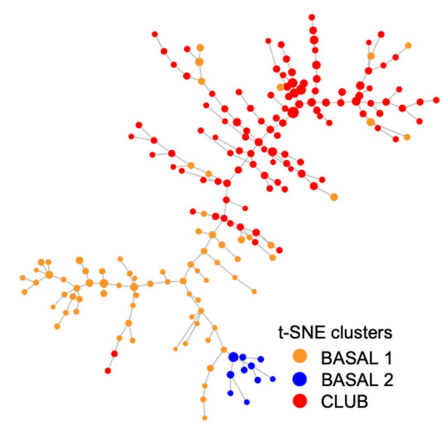

e

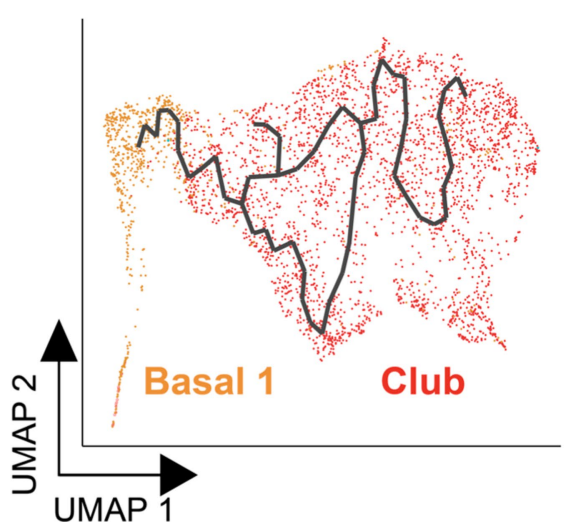

Extended Data Fig. 3 | Trajectory inference with SPADE and pseudotime. a, SPADE plot of pooled cells where each point represents cell states that are more related on the same or adjacent branches of a minimum spanning tree. Note: AT2 cells exist on a branch distal to basal and club cells, suggesting no lineage hierarchy between AT2, basal, and club cells. b, SPADE plots of pooled scRNA-seq samples after excluding AT2, VIM ${ }^{+}$and $H L A-D R^{+}$cells support lineage relationships between basal (blue) and club (red) populations by club cell branches emanating from basal cells. c, SPADE plots of Basal 1, Basal 2, and

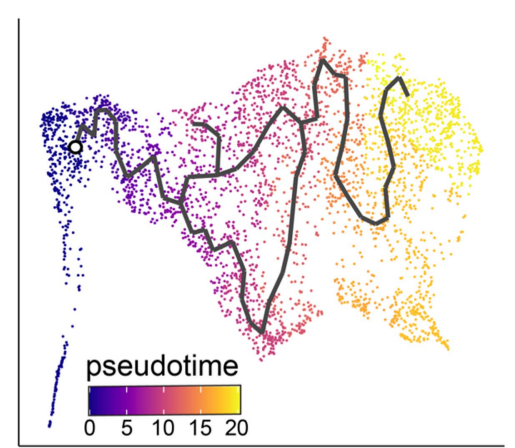

club populations. d, Left, gene expression of SCGB1A1 shows higher expression in club versus basal cell lineages (left) as compared to KRT5 (middle). Right, median gene expression of TNFRSF12A, showing a high (orange outline) and a low (blue outline) within basal cell branches and inferring a potential lineage relationship.e, Monocle 3 pseudotime trajectory analysis of single cell transcriptomes of Basal 1 connected to club cells depicted with UMAP (cell number, $n=3,721$ ), colored by (left) cell cluster and (right) pseudotime gradient. 
a
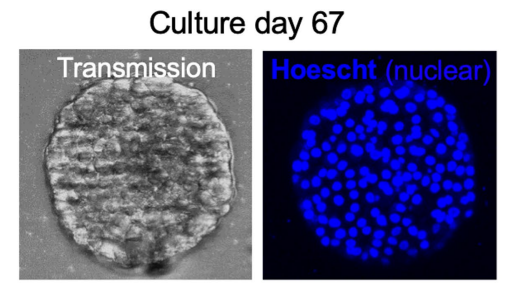

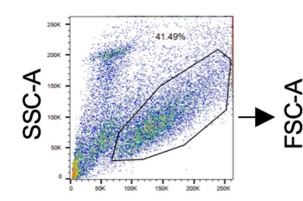

FSC-A

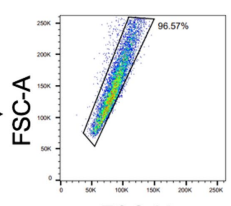

FSC-H

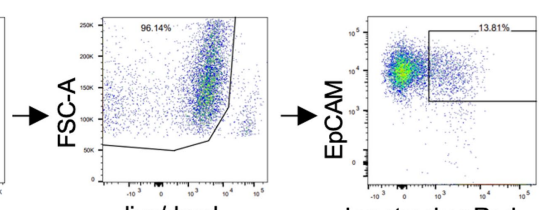

live/dead
Lysotracker Red
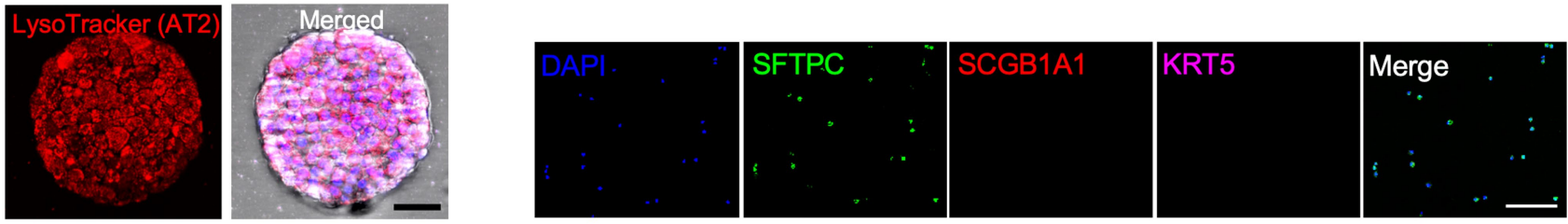

b

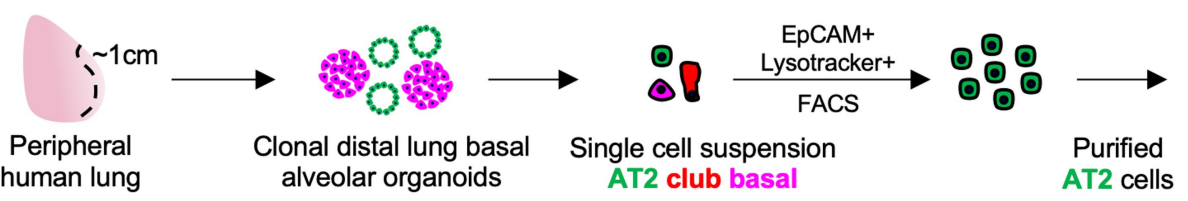

C

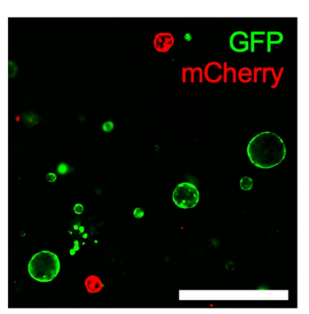

$\mathbf{f}$

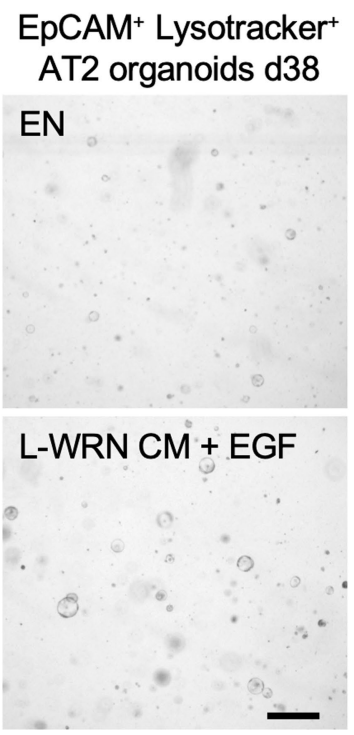

Extended Data Fig. 4 | Human AT2 organoid characterization. a, Left, confocal images of a live AT2 organoid at 67 days of culture labelled with Hoescht nuclear stain and LysoTracker Red DND-99. Top right, isolation of purified AT2 organoids. Representative FACS plots showing LysoTracker Red AT2 purification from unfractionated organoid cultures. Bottom right, immunostaining of cytospin of LysoTracker-sorted AT2 cells show high purity $\left(100 / 100\right.$ cells SPC ${ }^{+}$SCGB1A1 KRT5); scale bar $\left.=50 \mu \mathrm{m}\right) . \mathbf{b}$, Schema of FACS isolation of AT2 cells from human mixed distal lung organoids as EPCAM $^{+}$LysoTracker ${ }^{+}$AT 2 cells followed by long-term clonogenic organoid culture. c, Representative image of clonal mixing studies from stromadepleted, EPCAM ${ }^{+}$LysoTracker ${ }^{+}$, lentivirally-marked AT2 cells demonstrating presence of completely mCherry ${ }^{+}$or GFP $^{+}$but not chimaeric organoids carried out as in Extended Data Fig. 1e-g, passage 1 after lentiviral infection, scale bar $=200 \mu \mathrm{m} . \mathbf{d}$, Quantitation of red, green, or chimaeric AT2 organoid cultures as in (c) from two individuals $(1,2)$ after initial and serial passaging ( $\mathrm{P} 1=$ passage 1).e, AT2 organoid proliferation with differing combinations of recombinant niche factors and PORCUPINE inhibitor C59 (1 mM), NOGGIN (N), EGF (E), WNT3A (W), R-SPONDIN1 (R). $n=3$ per condition, data are mean \pm s.e.m., ${ }^{*}=P<0.05,{ }^{* * *}=P<0.001$, two-tailed student's $t$-test.f, Brightfield microscopy comparing pure AT 2 organoid growth enhancement in chemically defined lung organoid media (EN) versus serum-containing L-cell conditioned media containing WNT3A, NOGGIN, and R-SPONDIN3 (L-WRNCM) supplemented with recombinant EGF, one experiment. Scale bar $=200 \mu \mathrm{m}$. g, Transmission electron microscopy image of representative AT2 organoid at 28 days of culture. Note apical microvilli (black arrows) and lamellar bodies (red arrows); scale bar $=10 \mu \mathrm{m}$. 


\section{Article}

a

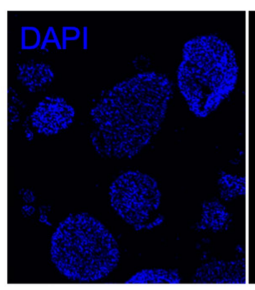

b

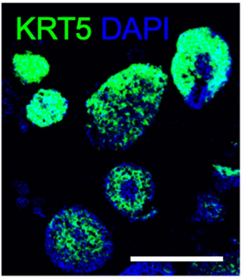

d

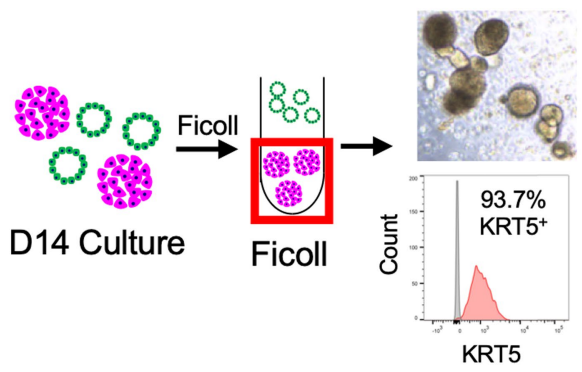

e

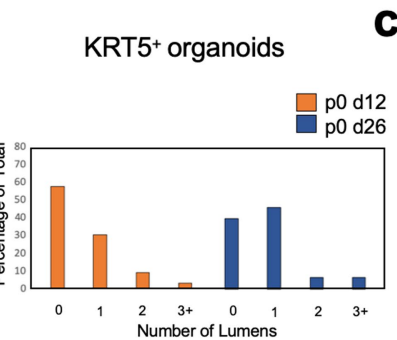

c

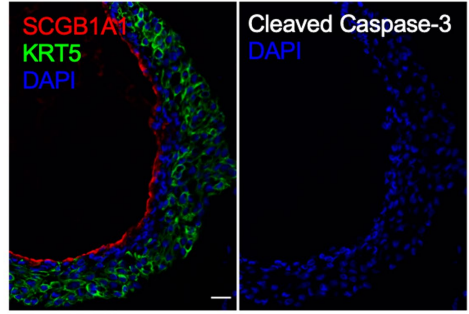

\section{Culture post-passage}

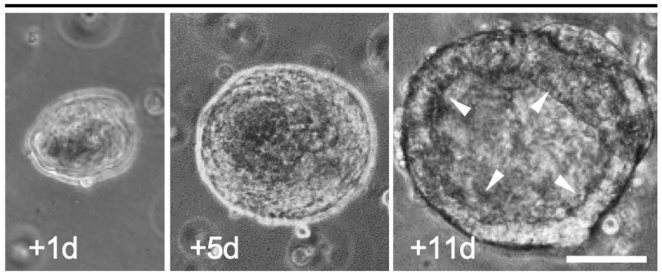

f

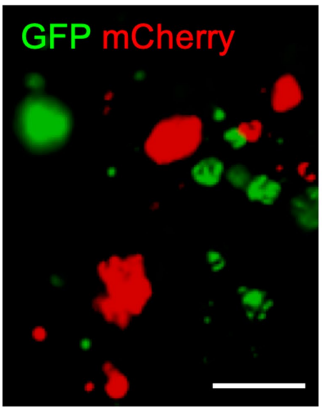

g

\begin{tabular}{|c|c|c|c|}
\hline & Red & Green & Chimera \\
\hline Lung 1 P0 & 114 & 107 & 0 \\
\hline Lung 1 P1 & 94 & 103 & 0 \\
\hline Lung 2 P0 & 104 & 86 & 0 \\
\hline Lung 2 P2 & 121 & 116 & 2 \\
\hline
\end{tabular}

h

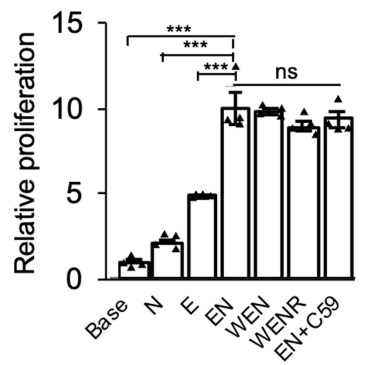

Extended Data Fig. 5 | Characterization of basal organoids. a-c, Basal organoids in mixed culture progressively form internal lumens, which is not associated with apoptosis. a, KRT5 IF, day 26 culture, scale bar $=200 \mu \mathrm{m}$. b. Lumen quantitation, d 12 versus $\mathrm{d} 26$ culture, single determination. c, Absence of apoptosis in $\mathrm{d} 26$ basal cell organoid internal lumen, cleaved caspase IF, from Fig. $2 \mathrm{~b}$, scale bar $=20 \mu \mathrm{m}$. d, e, Isolation of purified basal cell organoids via differential sedimentation in Ficoll. d, Schema and enrichment to $>90 \% \mathrm{KRT5}^{+}$cells as measured by intracellular KRT5 FACS of sedimented basal organoid cells; scale bar $=100 \mu \mathrm{m}$. e, Serial time lapse microscopy of sedimented basal organoids reveals spontaneous cavitation within two weeks post passage or within four weeks of culture initiation; scale bar $=25 \mu \mathrm{m}$. f, g, Clonal mixing studies from stroma-depleted, Ficoll-purified and lentivirally-marked basal organoid cells demonstrating fully mCherry ${ }^{+}$or GFP but not chimaeric organoids as in Extended Data Fig. 1f, passage 1 after lentiviral infection, scale bar $=200 \mu \mathrm{m}$.f, Representative clonal mixing image study. g, Quantitation. h, Growth factor evaluation for basal organoids after d14 sedimentation, enzymatic dissociation and clonogenic culture. Growth was not affected by the PORCUPINE inhibitor C59 $(1 \mu \mathrm{M}) . n=4$ per condition, data are mean \pm s.e.m., ${ }^{* * *}=P<0.001$, two-tailed student's $t$-test. 
a

Lung 1
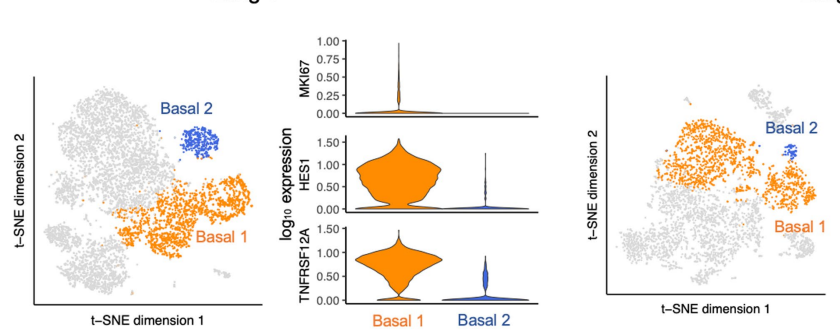

b

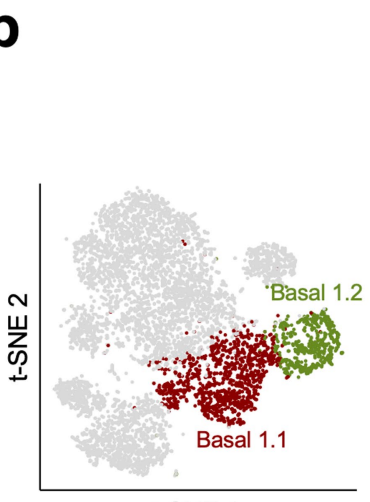

t-SNE 1

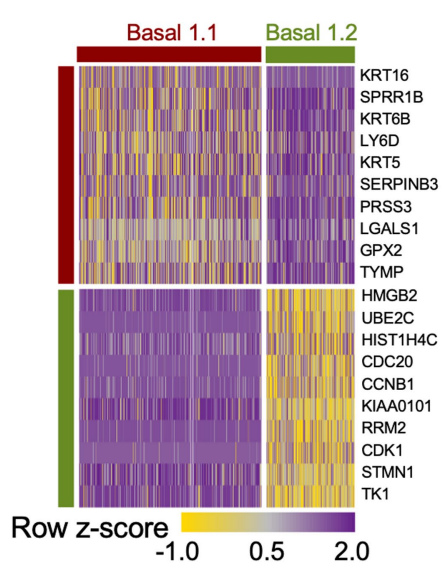

d
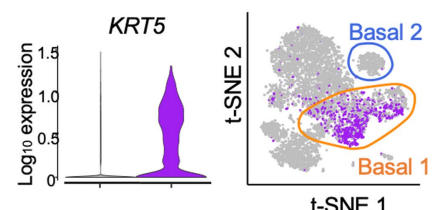

- EPCAM+ITGA6 ITGB4+ Basal 1 cells

remainder e

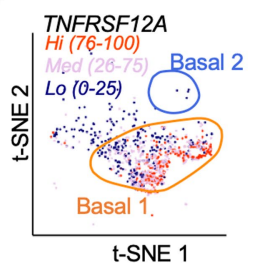

Extended Data Fig. 6 | scRNA-seq identifies an active basal cell subpopulation across three individual patient organoid cultures. a, High resolution clustering analysis identifies a reproducible active basal cell subpopulation with significantly higher expression of mRNAs for TNFRSF12A, the NOTCH pathway marker HES1, and the proliferation marker MKI67. Modified Kruskal-Wallis Rank Sum Test two tail p-values: TNFRSF12A 4.15× $10^{-8} ; H E S 12.4 \times 10^{-10} ; M K I 673.4 \times 10^{-3}$. b, Fine resolution clustering of KRT5 ${ }^{+}$ populations identifies two Basal 1 sub-clusters, Basal 1.1 and 1.2. c, Gene Ontology PANTHER overrepresentation of differentially expressed genes enriched in Basal 1.2 versus 1.1 show the majority of Basal 1.2 processes involve cell cycle (asterisks). Complete analysis is provided in Supplementary Table 3. d, Violin plot of scRNA-seq analysis for Fig. $2 \mathrm{f}-\mathrm{h}$ depicting KRT5 mRNA

Lung 2
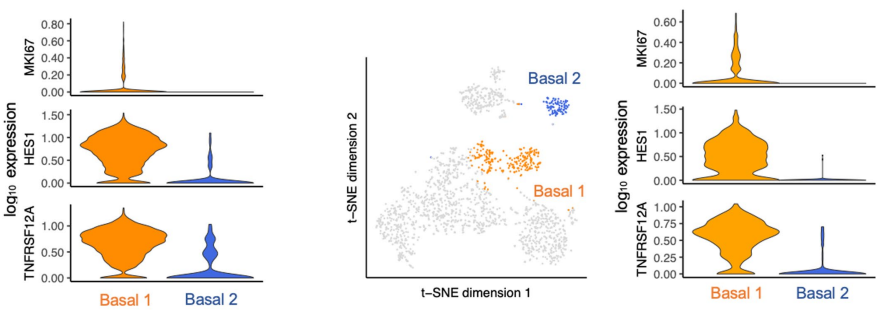

C

\begin{tabular}{|c|c|}
\hline \multicolumn{2}{|l|}{ GO Biological Process } \\
\hline cell cycle (GO:0007049)* & \\
\hline cell cycle process (GO:0022402) ${ }^{\star}$ & $9.39 \mathrm{E}-52$ \\
\hline mitotic cell cycle (GO:0000278)* & $4.21 \mathrm{E}-46$ \\
\hline mitotic cell cycle process (GO:1903047)* & $2.38 \mathrm{E}-41$ \\
\hline chromosome organization (GO:0051276)* & $2.38 \mathrm{E}-39$ \\
\hline cell division (GO:0051301)* & $3.21 \mathrm{E}-35$ \\
\hline organelle organization (GO:0006996) & $5.25 \mathrm{E}-32$ \\
\hline DNA metabolic process (GO:0006259) & 3.95E-26 \\
\hline cell cycle phase transition (GO:0044770)* & $1.69 \mathrm{E}-25$ \\
\hline chromosome segregation (GO:0007059) & $4.35 \mathrm{E}-25$ \\
\hline nuclear chromosome segregation (GO:0098813) & $6.20 \mathrm{E}-25$ \\
\hline mitotic cell cycle phase transition (GO:0044772)* & $1.03 \mathrm{E}-24$ \\
\hline sister chromatid segregation (GO:0000819) ${ }^{\star}$ & 3.15E-24 \\
\hline nuclear division (GO:0000280)* & $1.03 \mathrm{E}-21$ \\
\hline DNA replication (GO:0006260) & 2.44E-21 \\
\hline DNA conformation change (GO:0071103) & $2.78 \mathrm{E}-21$ \\
\hline organelle fission (GO:0048285) & $2.46 \mathrm{E}-20$ \\
\hline mitotic nuclear division (GO:0140014)* & 4.69E-20 \\
\hline cellular component organization (GO:00 & $5.50 \mathrm{E}-20$ \\
\hline regulation of cell cycle (GO:0051726) & $1.62 \mathrm{E}-19$ \\
\hline
\end{tabular}

$\mathbf{f}$

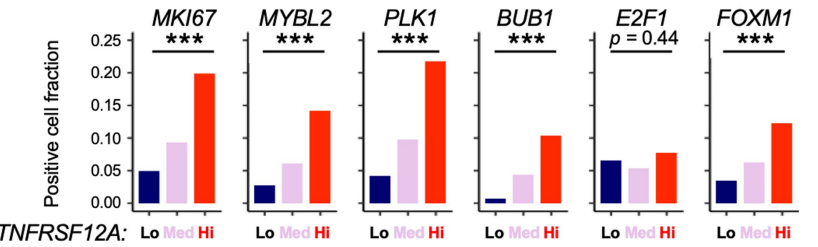

expression among triply $E P C A M^{+} I T G A 6^{+} I T G B 4^{+}$mRNA-expressing single cells (purple, that is, tandem mRNA expression of all three genes) versus the remainder of cells (grey), $P<0.001$ two tailed Kruskal-Wallis Rank Sum Test. e, $\mathrm{t}-\mathrm{SNE}$ visualization of TNFRSF12A and ITGA6 expression from d among cells with $E P C A M^{+} I T G A 6^{+} I T G B 4^{+}$gene expression and subdivision by high (top quartile, orange), medium (pink) and low (bottom quartile, navy blue) mRNA expression.f, Proliferation-associated gene expression is progressively enriched for scRNA-seq cell fractions of in $E P C A M^{+} I T G A 6^{+} I T G B 4^{+}$cells that are stratified for low, medium, or high expression of TNFRSF12A mRNA as in e. Data in $\mathbf{f}$ represent cell population fractions from a single experiment. ${ }^{* * *}=P<0.001$, two tailed Chi-square test. 
a

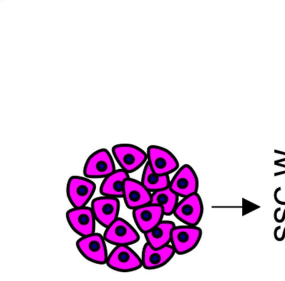

KRT5+ basal organoids d14 Ficoll-purified
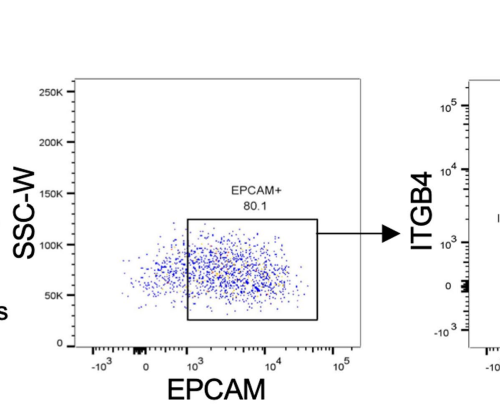

d

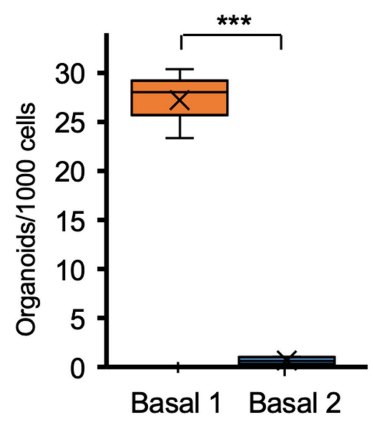

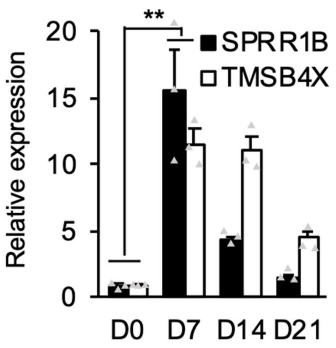

b

KRT5 intracellular FACS

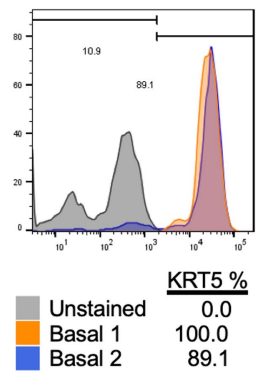

f

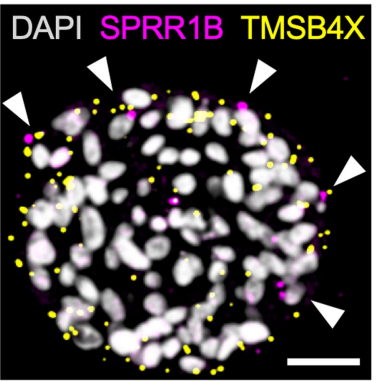

g

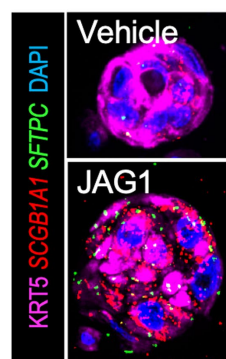

EPCAM+ITGA6+ITGB4+TNFRSF12A ${ }^{\text {hi }}$
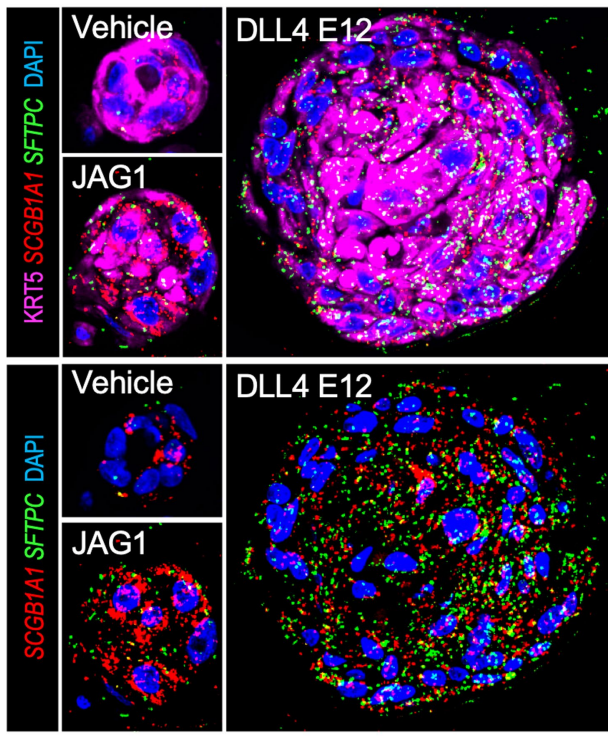

Extended Data Fig. 7 | Evaluation of Basal 1 lineage relationship to Basal 2 and the influence of NOTCH signalling on Basal 1 renewal and differentiation. a, Isolation of Basal 1 and Basal 2 via differential sedimentation of KRT5 ${ }^{+}$cells followed by FACS sorting of EPCAM ${ }^{+} \mathrm{ITGA}^{+}{ }^{+} \mathrm{ITGB} 4^{+} \mathrm{TNFRSF} 12 \mathrm{~A}^{+}$ (Basal 1) versus EPCAM ITGA6 ITGB4 TNFRSF12A (Basal 2). b, Intracellular FACS measurement of KRT5 protein expression in Basal 1 and 2 fractions from a. c, Representative brightfield of day 14 organoid cultures from $\mathbf{a}, \mathbf{b}$. d, Quantitation of 3 independent experiments from a-c, box plot represents first quartile, median, third quartile, and whiskers represent minimum and maximum. ${ }^{* * *} P<0.001$, two tailed student's $t$-test. e, qPCR measurement of two differentially upregulated Basal 2 genes from the three scRNA-seq biological replicates (Extended Data Fig. 6, SPRR1B, TMSB4X) after prolonged culture of FACS isolated Basal 1 cells. Data are relative mean \pm s.e.m. of cultures from three independent experiments, ${ }^{* *}=P<0.01$, two tailed student's $t$-test. h

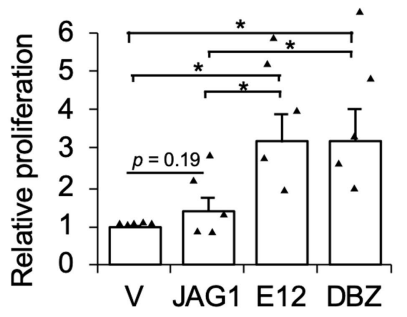

i

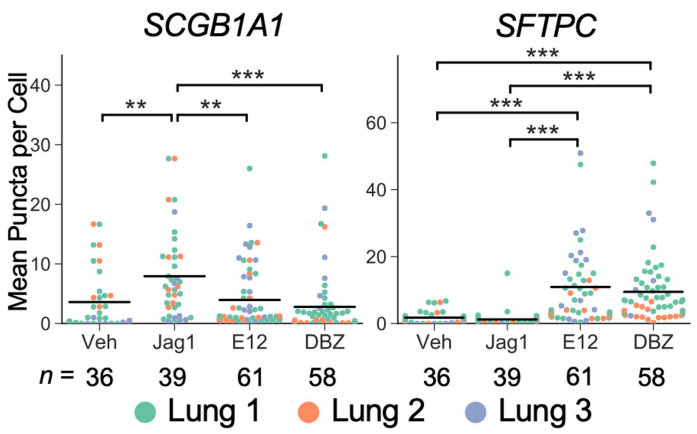

f, RNA FISH demonstrating TMSB4X and SPRR1B cellular transcripts within organoids originating from Basal 1 cells (arrows), scale bar $=25 \mu \mathrm{m}$. g, KRT5 immunostaining and SFTPC and SCGB1A1 RNA FISH of FACS isolated TNFRSF12A ${ }^{\text {hi }}$ Basal 1 cells under vehicle, NOTCH agonism (JAG1 peptide), or $\mathrm{NOTCH}$ antagonism with the Delta-like ligand mutant 4 (DLL4 ${ }^{\mathrm{E12}}$;E12) or the gamma secretase inhibitor DBZ; scale bar $=50 \mu \mathrm{m}$. h, Fluorescent quantitation of resazurin dye reduction to estimate relative cellular proliferation in $\mathbf{g}$, data are normalized to vehicle $(\mathrm{V})$ and represent mean \pm s.e.m. from five independent experiments, ${ }^{*} P<0.05$, two tailed student's $t$-test.i, Quantitation of SCGB1A1 and SFTPC gene expression by RNA FISH in the context of NOTCH agonism or antagonism from three independent experiments, ${ }^{* *} P<0.01$, ${ }^{* * *} P<0.001$, two tailed student's $t$-test. SFTPC mRNA upregulation was not accompanied by lamellar body or SFTPC protein production (data not shown). 

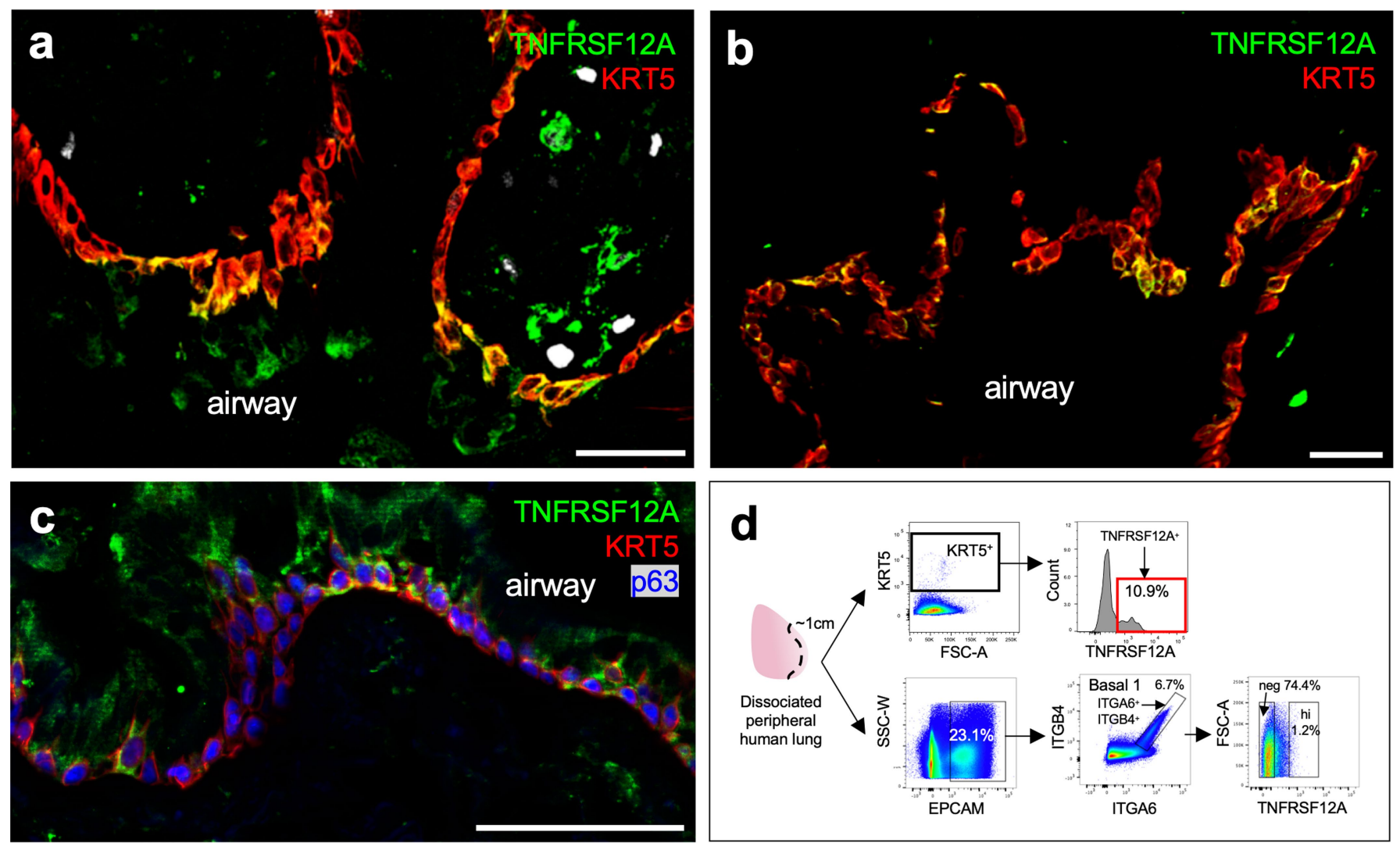

Extended Data Fig. 8 | TNFRSF12A marks a subset of distal airway basal cells in vivo. a, b, Immunostaining of KRT5 and TNFRSF12A in human distal airways from two individuals, scale bar $=100 \mu \mathrm{m}$. c, Immunostaining of KRT5, TNFRSF12A, and p63 in human distal airway, scale bar $=100 \mu \mathrm{m} . \mathrm{d}$, FACS

monoclonal anti-TNFRSF12A (cell surface) (top), or sequential FACS isolation from freshly dissociated human distal lung of EPCAM ${ }^{+} \mathrm{TGA6}^{+} \mathrm{ITGB} 4^{+}$cells followed by fractionation into TNFRSF12 $A^{\text {hi }}$ or TNFRSF12A ${ }^{\text {neg }}$ subsets (bottom), pre-gated on live singlets and used for culture experiments in Fig. 3d-g. 


\section{Article}

a
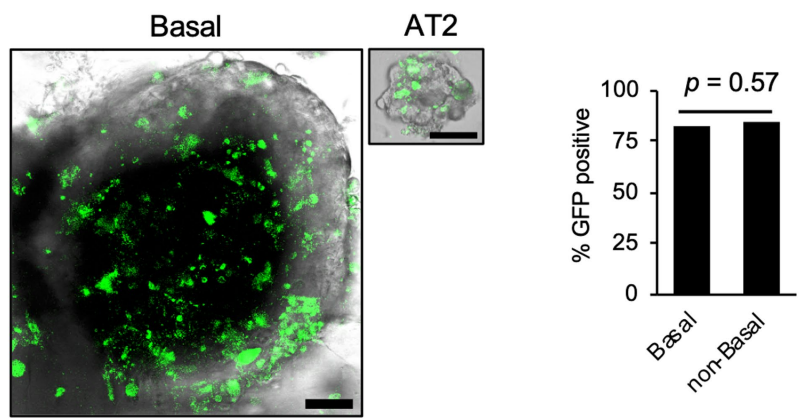

C
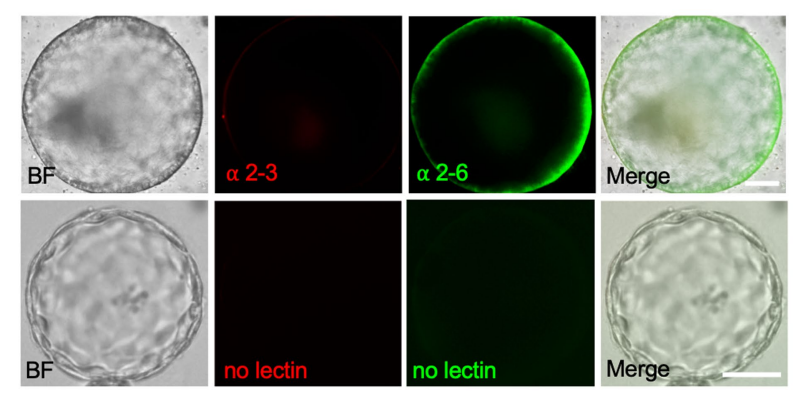

e

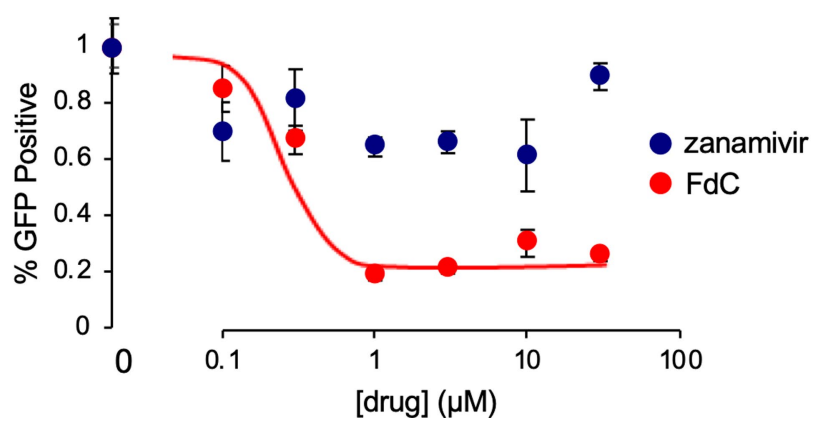

Extended Data Fig. 9 |Influenza infection modelling in mixed distal lung organoid cultures. a, b, Distal lung organoid modelling of $\mathrm{H} 1 \mathrm{~N} 1$ influenza infection. a, Merged transmission and GFP confocal images of purified basal (left) and purified AT2 organoids (right) $12 \mathrm{~h}$ after infection with PR8-GFP H1N1 influenza virus, quantified by FACS for $\% \mathrm{GFP}^{+}$cells. Bar plot represents the mean percentage of infected cells from three technical replicates, $P=0.57$, Chi-square test. Scale bars $=50 \mu \mathrm{m}$. b. Viral genome quantitation over time of mixed distal lung organoid culture supernatants subjected to initial infection of wild-type H1N1 at an estimated multiplicity of infection (MOI) of 0.01 , qRT-PCR, data represent the mean of three independent experiments \pm s.e.m. c, d, Lectin staining with M. amurensis (a2-3) and S. nigra (a2-6) lectins or no b

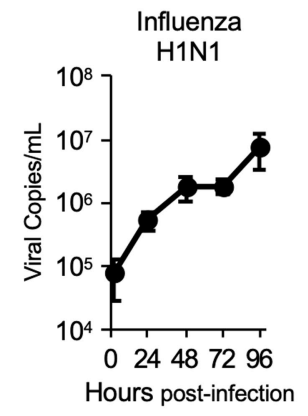

d
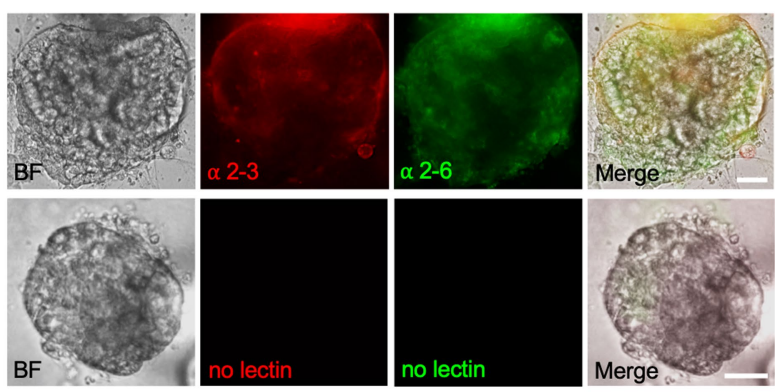

f

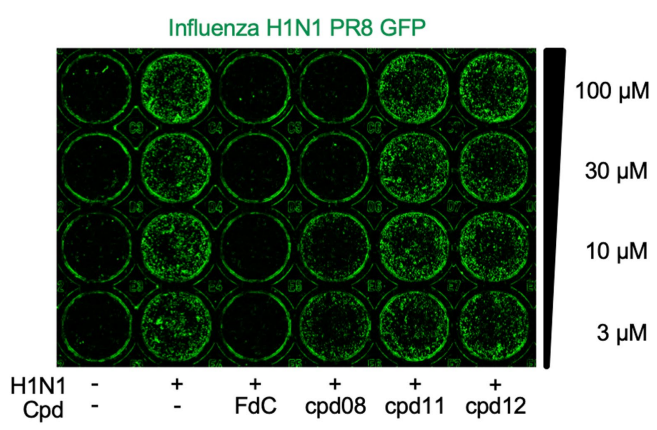

lectin negative controls to characterize sialic acid residues which serve as surface molecules for influenza virus host cell entry. AT2 organoids (c) and basal organoids (d). Scale bar $=25 \mu \mathrm{m}$. e, Dose response curves for two different classes of antiviral drugs on influenza infectivity and replication. The nucleoside analogue $\mathrm{FdC}$ demonstrated dose dependent activity with $\mathrm{IC}_{50}$ of $340 \mathrm{nM}$ as compared to neuraminidase inhibitor zanamivir, which only impairs viral shedding but not infectivity and replication. $n=3$ per condition, data represents mean \pm s.e.m. f, Fluorescence micrograph of multiwell screening of selected various antiviral agents after H1N1 PR8-GFP organoid infection in 48 well format. $\mathrm{FdC}=$ nucleoside analogue 2'-deoxy-2'-fluorocytidine. Cpd $=$ compound $\#$. 
a

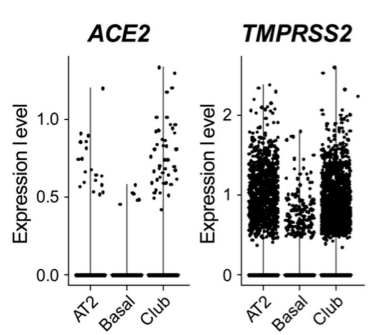

b

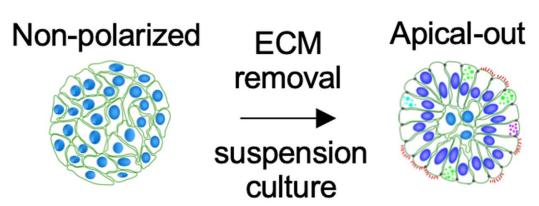

C

\section{Non-polarized cross section}

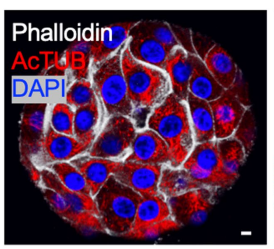

Apical-out cross section

\section{Eversion and accelerated ciliary differentiation of apical-out basal organoids}
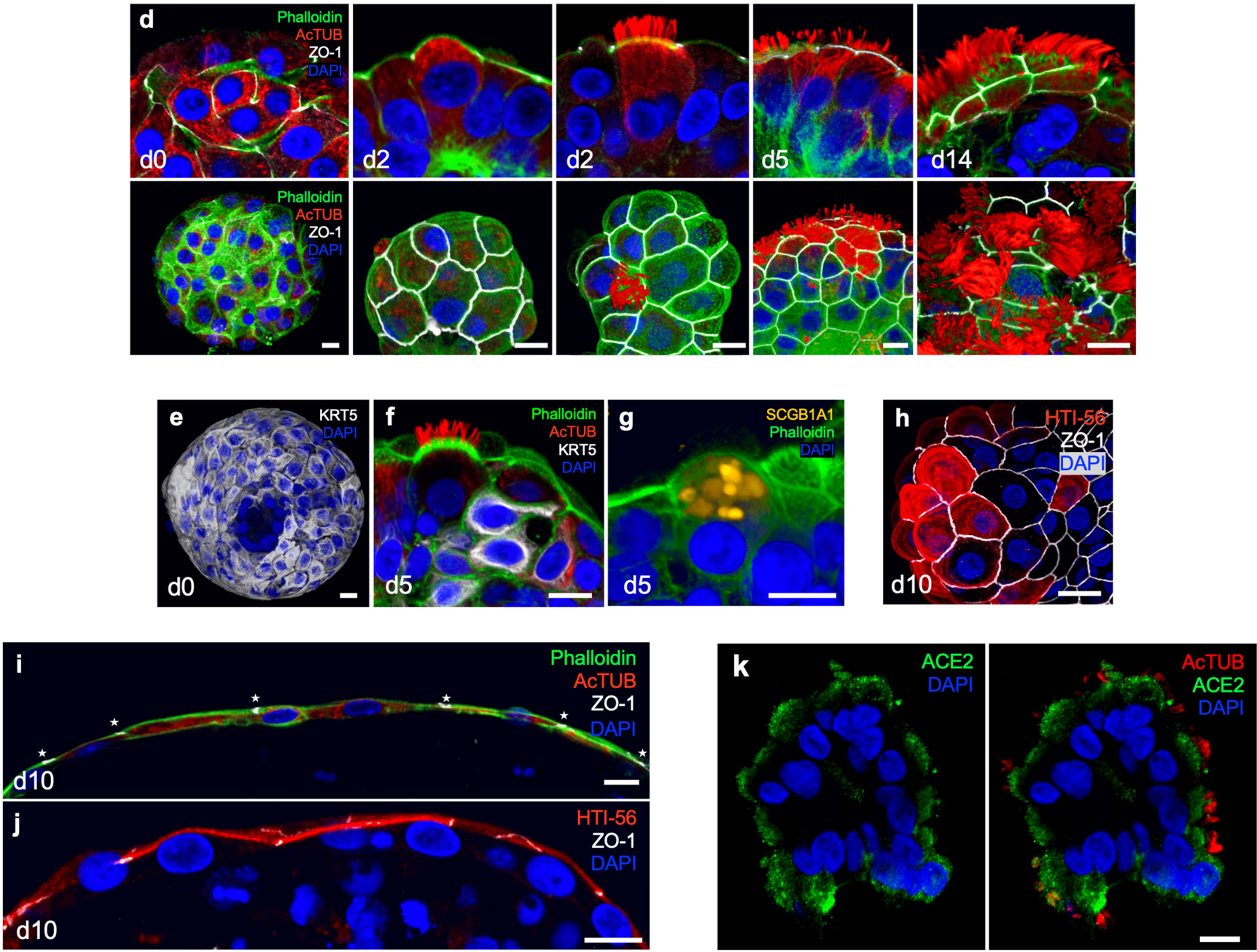

Extended Data Fig. 10 | Apical-out polarization and multi-lineage differentiation of distal lung organoids upon suspension culture. a, scRNA-seq plots of $A C E 2$ and TMPRSS2 gene expression in ECM-embedded mixed distal lung organoids as in Fig. 1a-h. b, Diagram of ECM removal and suspension culture leading to apical-out polarity of lung organoids. c, Representative confocal microscopy showing reorganization of microfilaments (phalloidin) and acetylated microtubules (AcTUB) upon ECM removal. Scale bar $=10 \mu \mathrm{m}$. d-f, Polarization and accelerated ciliary differentiation of apical-out basal organoids. d, Confocal 3D sections (top panels) and surface reconstructions (bottom panels) of apical-out lung organoids at different days after ECM removal. At day 0 (d0) microfilament (green, phalloidin) and microtubule (red, acetylated tubulin) organization is not polarized while junctional strands (ZO-1, white) are polarized. By day 2 in suspension (d2) ZO-1 (white) forms junctional rings in the apical periphery of each cell facing the external side of the organoids and the actin cytoskeleton forms microvilli (green) facing outward (apical-out polarity). Also, at d2 some cells initiate microtubule polarization. By day 5 (d5) many more cells have motile cilia facing outwards. Mature motile cilia can be observed for several weeks, example at day 14 (d14).e, 3D confocal reconstruction of an organoid embedded in ECM consisting mostly of basal stem cells (KRT5 ${ }^{+}$, white).f, As apical-out polarity is established in suspension culture and ciliogenesis begins, $\mathrm{KRT5}^{+}$basal cells are found underneath the polarized epithelium. g, SCGB1A1 ${ }^{+}$ Club cells with apical-out polarity are present on the exterior surface. In all panels nuclei are stained blue with DAPI, and actin microfilament organization visualized with phalloidin (green). Scale bars $=10 \mu \mathrm{m}$. h-j, Prolonged suspension culture of AT2 organoids (day 10 post-suspension) induces apical-out polarization and AT1 differentiation. h, Optical sections through alveolar-derived organoids after 10 days in suspension culture show decreased abundance of AT 2 cells while individual cuboidal cells begin to express the AT1 marker $\mathrm{HTI}-56$ (red), a transmembrane protein specific to the apical membrane of alveolar type 1 pneumocytes (AT1). Scale bars $=10 \mu \mathrm{m}$. $\mathbf{i}, \mathbf{j}$, Side views of alveolar organoids after 10 days of suspension culture reveal thin AT1 cells with phalloidin-reactive apical junctional complexes facing outwards (apical-out) (i) and expression of HTI-56 on the apical membrane (j). Scale bars $=10 \mu \mathrm{m}$. k, Representative confocal microscopy immunofluorescence of apical-out human basal organoid after 10 days in suspension expressing SARS-CoV-2 receptor ACE2 (green), cilia (AcTUB, red) and DAPI (blue). Scale bar $=20 \mu \mathrm{m}$. 


\title{
natureresearch
}

Corresponding author(s): Blish CA, Desai TJ, and Kuo CJ

Last updated by author(s): 11/17/2020

\section{Reporting Summary}

Nature Research wishes to improve the reproducibility of the work that we publish. This form provides structure for consistency and transparency in reporting. For further information on Nature Research policies, see Authors \& Referees and the Editorial Policy Checklist.

\section{Statistics}

For all statistical analyses, confirm that the following items are present in the figure legend, table legend, main text, or Methods section.

n/a Confirmed

$\square$ \ The exact sample size $(n)$ for each experimental group/condition, given as a discrete number and unit of measurement

$\square$ \A statement on whether measurements were taken from distinct samples or whether the same sample was measured repeatedly

$\triangle$ The statistical test(s) used AND whether they are one- or two-sided

Only common tests should be described solely by name; describe more complex techniques in the Methods section.

$\square$ \ A description of all covariates tested

$\square$ \A description of any assumptions or corrections, such as tests of normality and adjustment for multiple comparisons

$\square$ A full description of the statistical parameters including central tendency (e.g. means) or other basic estimates (e.g. regression coefficient)

$\triangle$ AND variation (e.g. standard deviation) or associated estimates of uncertainty (e.g. confidence intervals)

$\square$ For null hypothesis testing, the test statistic (e.g. $F, t, r$ ) with confidence intervals, effect sizes, degrees of freedom and $P$ value noted

$\square$ Give P values as exact values whenever suitable.

Х $\square$ For Bayesian analysis, information on the choice of priors and Markov chain Monte Carlo settings

Х $\square$ For hierarchical and complex designs, identification of the appropriate level for tests and full reporting of outcomes

Х $\square$ Estimates of effect sizes (e.g. Cohen's $d$, Pearson's $r$ ), indicating how they were calculated

Our web collection on statistics for biologists contains articles on many of the points above.

\section{Software and code}

Policy information about availability of computer code

Data collection scRNA-seq data was generated from three primary distal lung organoid cultures and one purified AT2 organoid culture using the 10X Chromium Single Cell 3' droplet based capture platform. Libraries were sequenced on an Illumina NextSeq 500. Data collection, sample multiplexing, barcode processing, RNA-seq alignment, and 3' gene counting were performed with Cell Ranger (version 1.1).

Data analysis

\begin{abstract}
Source code for single cell analysis is available at https://github.com/ameen-salahudeen/lung organoid. The Cell Profiler (version 2.0) pipeline used for RNA Proximity Ligase quantitation is available at https://github.com/tischi/cellprofiler-practical-NeuBIAS-Lisbon-2017 ImageJ (version 2.0) pipeline used for organoid counting is described in Papadopulos et al. Ultrastruct Pathol. 31:401-7 (2007).
\end{abstract}

For manuscripts utilizing custom algorithms or software that are central to the research but not yet described in published literature, software must be made available to editors/reviewers. We strongly encourage code deposition in a community repository (e.g. GitHub). See the Nature Research guidelines for submitting code \& software for further information.

\section{Data}

Policy information about availability of data

All manuscripts must include a data availability statement. This statement should provide the following information, where applicable:

- Accession codes, unique identifiers, or web links for publicly available datasets

- A list of figures that have associated raw data

- A description of any restrictions on data availability

Source Data are provided with this manuscript for Figures 2, 3 and Extended Data Figures 4, 5, 7 and 9. scRNA-seq data can be accessed on Gene Expression Omnibus under accession number GSE106850 (https://www.ncbi.nlm.nih.gov/geo/query/acc.cgi?acc=GSE106850). All data are available from the authors upon reasonable request. 


\section{Field-specific reporting}

Please select the one below that is the best fit for your research. If you are not sure, read the appropriate sections before making your selection. $\bigotimes$ Life sciences $\quad \square$ Behavioural \& social sciences $\square$ Ecological, evolutionary \& environmental sciences

For a reference copy of the document with all sections, see nature.com/documents/nr-reporting-summary-flat.pdf

\section{Life sciences study design}

All studies must disclose on these points even when the disclosure is negative.

Sample size No sample size calculations were performed. A minimum of 3 biological replicates was chosen for experiments for hypothesis testing against control groups to have at least two degrees of freedom. Sample sizes in scRNA-seq analysis were based on unsupervised clustering of all available viable cells - i.e. no downsampling, including all cells annotated for a given cluster. The scRNA-seq analysis was exploratory, and cell populations far exceeded what was anticipated as necessary for major cell type annotation ( $n>100$ cells). scRNA-seq experiments for distal lung organoid cultures were repeated three times for the purposes of reproducibility.

Data exclusions Data was excluded when organoid cultures exhibited microbial contamination.

Replication Biological replicates represent organoid cultures from unique individuals i.e. an experiment was performed on an organoid derived from individual 1, and reproduced in cultures derived from individuals 2, 3, etc. Images or experiments showing technical replicates is representative of at least two experiments unless otherwise specified. Single cell RNA-seq analysis was performed on organoid cultures derived from three individuals. All replication attempts were successful.

Randomization Experiments were performed on organoid cultures sequentially as they reached time points specified for each experiment. Control groups and experimental groups were paired for each culture. Randomization or control of covariates was not relevant as each experimental replicate used the same patient derived culture for both the experimental and control group.

Blinding Experiments involving more than one group utilized blinding as follows: Organoid formation counting was performed by two independent observers one of whom was blinded to the group assignments or was performed using automated image pipelines with standardized parameters across all assigned groups for raw output. The Cell Profiler pipeline used for RNA Proximity Ligase quantitation is available at https://github.com/tischi/cellprofiler-practical-NeuBIAS-Lisbon-2017. The ImageJ pipeline used for organoid counting is described in Papadopulos et al. Ultrastruct Pathol. 31:401-7 (2007). Data was analyzed in a blinded manner.

\section{Reporting for specific materials, systems and methods}

We require information from authors about some types of materials, experimental systems and methods used in many studies. Here, indicate whether each material, system or method listed is relevant to your study. If you are not sure if a list item applies to your research, read the appropriate section before selecting a response.

Materials \& experimental systems

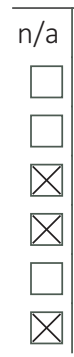

$\mathrm{n} / \mathrm{a}$ Involved in the study

$\bigotimes$ Antibodies

\ Eukaryotic cell lines

$\bigotimes \square$ Palaeontology

Х Animals and other organisms

$\bigotimes$ Human research participants

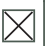
Clinical data

\begin{tabular}{l|l}
\multicolumn{2}{l}{ Methods } \\
\hline n/a & Involved in the study \\
$\square$ & $\square$ ChIP-seq \\
$\square$ & $\bigotimes$ Flow cytometry \\
$\square$ & $\square$ MRI-based neuroimaging
\end{tabular}

\section{Antibodies}

Antibodies used

Mouse anti- HTII-280, Terrace Biotech, TB-27AHT2-280; Mouse anti-HTI-56, Terrace Biotech, TB-29AHT1-56; Rabbit anti- SFTPC, Santa Cruz Biotechnology, sc-13979; Mouse anti-acetyl- $\alpha$-tubulin, Sigma Aldrich, T7451; Rabbit anti- Cytokeratin 5, AbCam, Ab193895; Mouse anti- SCGB1A1, Santa Cruz, sc-365992; Goat anti-ACE2, AbCam, AF933; Sheep anti-SCGB1A1, Novus, AF4218; Rabbit anti-ZO-1, Invitrogen, 617300; Mouse anti- SARS-CoV2 NP, Sinobiological, 40143-MM05; Mouse anti-dsRNA, Scicons, 10010200; Rabbit anti-acetyl- $\alpha$-tubulin, Cell Signaling, 5335; Mouse anti-TNFRSF12A, Biolegend, 314102; Rabbit anti-TNFRSF12A, Thermo Fisher, PA5-20275, lot TD2561104.

Validation

All antibodies were validated by the manufacturer with additional citations in the primary literature. The ITEM-4 clone of TNFRSF12A was validated in the literature in knockout-mouse studies and those are cited in the manufacturer website and also in the Validated Antibody Database (https://www.labome.com/knockout-validated-antibodies/ANTI-Fn14-antibody-knockoutvalidation-BioLegend-314102.html). ITEM-4 was also validated with qPCR as shown in Supplementary Figure 1. ITEM-4 clone was used to validate a second rabbit polyclonal antibody (ThermoFisher PA5-20275) as determined by dual staining and confirmation of overlap by FACS and immunofluorescence of organoid and tissue cryosections. 
Policy information about cell lines

Cell line source(s)

Primary organoid cultures were generated from human lung biospecimens. Vero E6 cells used in this study were thawed directly from a cryopreserved aliquot purchased from ATCC.

Authentication

Vero E6 cells were thawed direcly from a cryopreserved aliquot purchased from ATCC.

Mycoplasma contamination

All human biospecimens were definitively treated with the anti-mycoplasma antibiotic normocin during primary plating. Mycoplasma surveillance was performed on cultures on an ad-hoc basis and mycoplasma was never detected. Vero E6 cells (ATCC CRL-1586) were purchased from ATCC which certified the lot to be free of Mycoplasma contamination.

Commonly misidentified lines (See ICLAC register)

No misidentified cell lines were used in this study

\section{Human research participants}

Policy information about studies involving human research participants

Population characteristics

Human subjects over the age of 18 were screened for informed consent irrespective of age, gender, or other demographic characteristic. Covariate relevant population characteristics are age, gender, anatomic location of lung specimen, cancer diagnosis, cancer staging, smoking status and pack year smoking history.

Recruitment

Participants were recruited if they were to undergo a surgical procedure involving resection of lung tissue and the recruitment population was biased for patients who were fit for surgery and who did not have known diagnoses of active HIV, HBV, HCV, or other known blood borne pathogens. This bias is very unlikely to impact experimental results, given the in vitro environment and cell autonomous nature of the study phenomenon.

Ethics oversight

All experiments were approved by the Stanford University IRB and followed all relevant guidelines and regulations. Informed consent was obtained from all participants

Note that full information on the approval of the study protocol must also be provided in the manuscript.

\section{Flow Cytometry}

\section{Plots}

Confirm that:

Х The axis labels state the marker and fluorochrome used (e.g. CD4-FITC).

ХThe axis scales are clearly visible. Include numbers along axes only for bottom left plot of group (a 'group' is an analysis of identical markers).

\All plots are contour plots with outliers or pseudocolor plots.

\A numerical value for number of cells or percentage (with statistics) is provided.

\section{Methodology}

Sample preparation

See methods and supplementary methods for full details. TrypLE digestion was performed for organoid culture and elastase digestion was performed for human distal lung.

Instrument

\section{BD FACS Aria Fusion}

Software

Data was collected using BD FACS Diva and analyzed using FlowJo version 10.0.8.

Cell population abundance

Post-sort fraction purity was confirmed by fixing and staining of cells with antibodies independent of the FACS panel as described in Extended Data Fig. 4 and 7. Specifically, 100/100 SFTPC+ DAPI nuclei for AT2 FACS purification, and 100\% KRT5+ cells for Basal 1 FACS purification.

Gating strategy

Flow cytometry data was first gated according to scatter properties, viability, then positive populations were identified using fluorescence minus one controls. Gating strategies are provided in the Supplementary Fig. 1.

\ Tick this box to confirm that a figure exemplifying the gating strategy is provided in the Supplementary Information. 